\title{
KELT-19Ab: A $P \sim$ 4.6-day Hot Jupiter Transiting a Likely Am Star with a Distant Stellar Companion
}

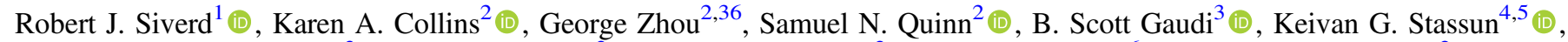

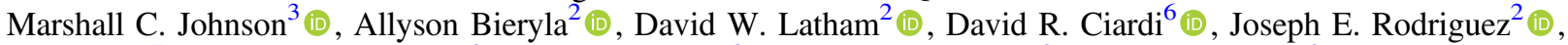

Kaloyan Penev ${ }^{7}$ (D), Marc Pinsonneault ${ }^{3}$ (1) Joshua Pepper $^{8}$ (10), Jason D. Eastman ${ }^{2}$ (iD, Howard Relles ${ }^{2}$, John F. Kielkopf ${ }^{9}$ (1), Joao Gregorio ${ }^{10}$, Thomas E. Oberst ${ }^{11}$, Giulio Francesco Aldi ${ }^{12,13}$, Gilbert A. Esquerdo ${ }^{2}$, Michael L. Calkins ${ }^{2}$ (1), Perry Berlind ${ }^{2}$, Courtney D. Dressing ${ }^{14,15,37}$ (1), Rahul Patel ${ }^{16}$ (1) , Daniel J. Stevens ${ }^{3}$, Thomas G. Beatty ${ }^{17,18}$ (1) , Michael B. Lund ${ }^{4}$ (D), Jonathan Labadie-Bartz ${ }^{8}$ (1), Rudolf B. Kuhn ${ }^{19,20}$, Knicole D. Colón ${ }^{21}$ (1) , David James ${ }^{22}$ (1) Xinyu Yao ${ }^{8}$, John A. Johnson ${ }^{2}$, Jason T. Wright ${ }^{17,18}$ (i), Nate McCrady ${ }^{23}$, Robert A. Wittenmyer ${ }^{24}$ (i), Samson A. Johnson ${ }^{3}$ (i), David H. Sliski ${ }^{25}$, Eric L. N. Jensen ${ }^{26}$ (10), David H. Cohen ${ }^{26}$ (1) Kim K. McLeod ${ }^{27}$ (10), Matthew T. Penny ${ }^{3,38}$ (1) , Michael D. Joner ${ }^{28}$, Denise C. Stephens ${ }^{28}$, Steven Villanueva, Jr. ${ }^{3}$ (1), Roberto Zambelli ${ }^{29}$, Christopher Stockdale ${ }^{30}$ (1), Phil Evans ${ }^{31}$,

Thiam-Guan $\operatorname{Tan}^{32}$ (1), Ivan A. Curtis ${ }^{33}$, Phillip A. Reed ${ }^{34}$, Mark Trueblood ${ }^{35}$, and Patricia Trueblood ${ }^{35}$

${ }^{1}$ Las Cumbres Observatory, 6740 Cortona Drive, Suite 102, Goleta, CA 93117, USA

${ }^{2}$ Harvard-Smithsonian Center for Astrophysics, Cambridge, MA 02138, USA; kcollins@cfa.harvard.edu

${ }^{3}$ Department of Astronomy, The Ohio State University, 140 West 18th Avenue, Columbus, OH 43210, USA

${ }^{4}$ Department of Physics and Astronomy, Vanderbilt University, Nashville, TN 37235, USA

${ }^{5}$ Department of Physics, Fisk University, 1000 17th Avenue North, Nashville, TN 37208, USA

${ }^{6}$ NASA Exoplanet Science Institute/Caltech, Pasadena, CA, USA

${ }^{7}$ Department of Physics, The University of Texas at Dallas, 800 West Campbell Road, Richardson, TX 75080-3021, USA

${ }^{8}$ Department of Physics, Lehigh University, 16 Memorial Drive East, Bethlehem, PA, 18015, USA

${ }^{9}$ Department of Physics and Astronomy, University of Louisville, Louisville, KY 40292, USA

${ }^{10}$ Atalaia Group \& CROW Observatory, Portalegre, Portugal

${ }^{11}$ Department of Physics, Westminster College, New Wilmington, PA 16172, USA

${ }^{12}$ Dipartimento di Fisica "E. R. Caianiello," Università di Salerno, Via Giovanni Paolo II 132, I-84084 Fisciano (SA), Italy

${ }^{13}$ Istituto Nazionale di Fisica Nucleare, Sezione di Napoli, I-80126 Napoli, Italy

${ }^{14}$ Division of Geological \& Planetary Sciences, California Institute of Technology, Pasadena, CA 91125, USA

${ }^{15}$ Department of Astronomy, University of California, Berkeley, CA 94720-3411, USA

${ }^{16}$ IPAC, Mail Code 100-22, Caltech, 1200 E. California Boulevard, Pasadena, CA 91125, USA

${ }^{17}$ Department of Astronomy \& Astrophysics, The Pennsylvania State University, 525 Davey Lab, University Park, PA 16802, USA

${ }^{18}$ Center for Exoplanets and Habitable Worlds, The Pennsylvania State University, 525 Davey Lab, University Park, PA 16802, USA

${ }^{19}$ South African Astronomical Observatory, P.O. Box 9, Observatory, 7935 Cape Town, South Africa

${ }^{20}$ Southern African Large Telescope, P.O. Box 9, Observatory, 7935 Cape Town, South Africa

${ }^{21}$ NASA Goddard Space Flight Center, Greenbelt, MD 20771, USA

${ }^{22}$ Astronomy Department, University of Washington, Box 351580, Seattle, WA 98195, USA

${ }^{23}$ Department of Physics and Astronomy, University of Montana, Missoula, MT 59812, USA

${ }^{24}$ University of Southern Queensland, Computational Engineering and Science Research Centre, Toowoomba, Queensland 4350, Australia

${ }^{25}$ Department of Physics and Astronomy, University of Pennsylvania, Philadelphia, PA 19104, USA

${ }^{26}$ Department of Physics and Astronomy, Swarthmore College, Swarthmore, PA 19081, USA

${ }^{27}$ Department of Astronomy, Wellesley College, Wellesley, MA 02481, USA

${ }^{28}$ Department of Physics and Astronomy, Brigham Young University, Provo, UT 84602, USA

${ }^{29}$ Società Astronomica Lunae, Castelnuovo Magra I-19030, Italy

${ }_{31}$ Hazelwood Observatory, Churchill, Victoria, Australia

${ }_{32}^{31}$ El Sauce Observatory, Coquimbo Province, Chile

${ }^{32}$ Perth Exoplanet Survey Telescope, Perth, Australia

${ }^{33}$ ICO, Adelaide, South Australia

${ }^{34}$ Department of Physical Sciences, Kutztown University, 15200 Kutztown Road, Kutztown, PA, 19530, USA

35 Winer Observatory, 22 Milky Way, Sonoita, AZ 85637, USA

Received 2017 September 19; revised 2017 November 20; accepted 2017 November 27; published 2017 December 22

\begin{abstract}
We present the discovery of the giant planet KELT-19Ab, which transits the moderately bright $(V \sim 9.9)$ A8V star TYC 764-1494-1 with an orbital period of 4.61 days. We confirm the planetary nature of the companion via a combination of radial velocities, which limit the mass to $\lesssim 4.1 M_{\mathrm{J}}(3 \sigma)$, and a clear Doppler tomography signal, which indicates a retrograde projected spin-orbit misalignment of $\lambda=-179.7_{-3.8}^{+3.7}$ degrees. Global modeling indicates that the $T_{\text {eff }}=7500 \pm 110 \mathrm{~K}$ host star has $M_{\star}=1.62_{-0.20}^{+0.25} M_{\odot}$ and $R_{\star}=1.83 \pm 0.10 R_{\odot}$. The planet has a radius of $R_{P}=1.91 \pm 0.11 R_{\mathrm{J}}$ and receives a stellar insolation flux of $\sim 3.2 \times 10^{9} \mathrm{erg} \mathrm{s}^{-1} \mathrm{~cm}^{-2}$, leading to an inferred equilibrium temperature of $T_{\text {eq }} \sim 1935 \mathrm{~K}$ assuming zero albedo and complete heat redistribution. With a $v \sin I_{*}=84.8 \pm 2.0 \mathrm{~km} \mathrm{~s}^{-1}$, the host is relatively slowly rotating compared to other stars with similar effective temperatures, and it appears to be enhanced in metallic elements but deficient in calcium, suggesting that it is likely an Am star. KELT-19A would be the first detection of an Am host of a transiting planet of which we are aware.
\end{abstract}

\footnotetext{
36 Hubble Fellow.

37 NASA Sagan Fellow.

${ }^{38}$ Sagan Fellow.
} 
Adaptive optics observations of the system reveal the existence of a companion with late-G9V/early-K1V spectral type at a projected separation of $\approx 160 \mathrm{au}$. Radial velocity measurements indicate that this companion is bound. Most Am stars are known to have stellar companions, which are often invoked to explain the relatively slow rotation of the primary. In this case, the stellar companion is unlikely to have caused the tidal braking of the primary. However, it may have emplaced the transiting planetary companion via the Kozai-Lidov mechanism.

Key words: methods: observational - planets and satellites: detection - planets and satellites: gaseous planets techniques: photometric - techniques: radial velocities - techniques: spectroscopic

Supporting material: data behind figures

\section{Introduction}

The Kilodegree Extremely Little Telescope (KELT; Pepper et al. 2003, 2007, 2012) survey was originally designed to discover transiting planets orbiting bright $\left(8 \leqslant V_{\text {mag }} \leqslant 11\right)$ host stars. The scientific value and strategy behind that approach was described in detail in the introduction of the recent discovery of KELT-20b (Lund et al. 2017). ${ }^{39}$ In short, these bright systems are the most amenable to detailed follow-up characterization (transit spectroscopy, secondary eclipse spectroscopy, phase curve measurements, etc.; Winn et al. 2010). Because the KELT project did not actively start to vet candidates until 2011, many of the initial transit candidates had already been discovered by other collaborations (e.g., Alonso et al. 2004; McCullough et al. 2006; Bakos et al. 2007; Collier Cameron et al. 2007).

This fact, combined with a few additional, coincidental, and nearly simultaneous occurrences, such as the confirmation of WASP-33b (Collier Cameron et al. 2010) via Doppler tomography (see Section 2.4.4 for an overview of this technique), our somewhat fortuitous discovery of KELT-1b (Siverd et al. 2012), and the "late entry" of KELT into the field of exoplanet discovery via transits, led us to pursue the discovery of transiting planets around hotter stars. This strategy has ultimately proven quite successful. In retrospect, the pursuit of hot stars was well suited to the survey, both because KELT observes a larger fraction of hot stars than other ground-based transit surveys (due to Malmquist bias, see Bieryla et al. 2015), but also because the reduction pipeline of the primary followup radial velocity vetting resource used by KELT, the Tillinghast Reflector Echelle Spectrograph (TRES) on the $1.5 \mathrm{~m}$ telescope at the Fred Lawrence Whipple Observatory, Mount Hopkins, Arizona, USA, was actively optimized to measure radial velocities of hot, rapidly rotating stars (Latham et al. 2009).

To date, this strategy of targeting hot stars has led to the discovery of four planets transiting A stars by the KELT survey: KELT-17b (Zhou et al. 2016b), KELT-9b (Gaudi et al. 2017), KELT-20b/MASCARA-2b (Lund et al. 2017; Talens et al. 2017b), and KELT-19Ab, the planet announced here. Additionally, there are four planets known to transit A stars discovered by other collaborations: WASP-33b (Collier Cameron et al. 2010), Kepler-13Ab (Shporer et al. 2011), HAT-P-57b (Hartman et al. 2015), and MASCARA-1b (Talens et al. 2017a).

As discussed in previous KELT planet discovery papers, rapidly rotating, hot stars above the Kraft break (Kraft 1967) pose unique challenges but provide unique opportunities. Transiting planets orbiting these stars are difficult to confirm

\footnotetext{
39 See also Talens et al. (2017b) for the simultaneous discovery of the same planet, MASCARA-2b.
}

via Doppler reflex motion, but on the other hand are amenable to Doppler tomography due to the large $v \sin I_{*}$ of their hosts.

It is also the case that A stars have a remarkable diversity in their properties, partially because their outer envelopes are primarily radiative, but exhibit extremely thin helium and hydrogen convective layers at the very outer edges of their atmospheres. In particular, the thin surface convection zones and very low mass-loss rates of A stars lead to very efficient gravitational settling of some elements, similar to (although not as extreme as) the settling exhibited in white dwarfs. This results in weaker spectral lines of those elements relative to what would be expected of a star of similar temperature, and not indicative of an actual global underabundance of those elements. Similarly, because the convective zones are so thin, partially ionized elements with large radiative cross sections below the convective zone can exhibit radiative levitation. This may lead to stronger lines that may be interpreted as large selective overabundances in certain elements (see, e.g., Richer et al. 2000). Indeed, it is even possible to have an element experience both gravitational settling and radiative levitation in different layers, creating a zone within an A star where that element is highly concentrated. In the case of iron, this effect may be severe enough to induce convective mixing, which can impact surface abundances (Richard et al. 2001).

In general, thinner surface convection zones that are lower in density experience gravitational settling at a faster rate, and are more susceptible to radiative levitation. In normal A stars, there are thin hydrogen and helium ionization zones that are very close to each other, which through overshoot behave as a single deeper mixed layer. However, if the helium ionization zone is driven much deeper and no longer in causal contact with the hydrogen ionization zone, even more extreme abundance changes may be apparent, since the hydrogen ionization zone by itself is isolated and very shallow.

The net result is that determining the global metal abundances for A stars can be extremely difficult. Abundances determined by atmospheric spectroscopy may have very little to do with the global metallic abundance of the star. A particularly notable example is the metallic-line Am stars (Titus \& Morgan 1940), which, although they have hydrogen lines consistent with the effective temperatures of late-A stars, also have metallic lines of heavier elements with strengths expected for cooler F stars, and lines of lighter elements consistent with hotter A stars. These Am stars are generally more slowly rotating (Abt \& Morrell 1995) than chemically normal stars with the same effective temperatures, likely due to a competition between elemental segregation and rotational mixing. The net result is that surface abundance anomalies can be enhanced in some elements and suppressed in others for Am stars (Abt \& Morrell 1995). Empirically, stars with rotational speeds above $\sim 150 \mathrm{~km} \mathrm{~s}^{-1}$ are chemically "normal" and it appears that mixing overcomes the settling described above. 


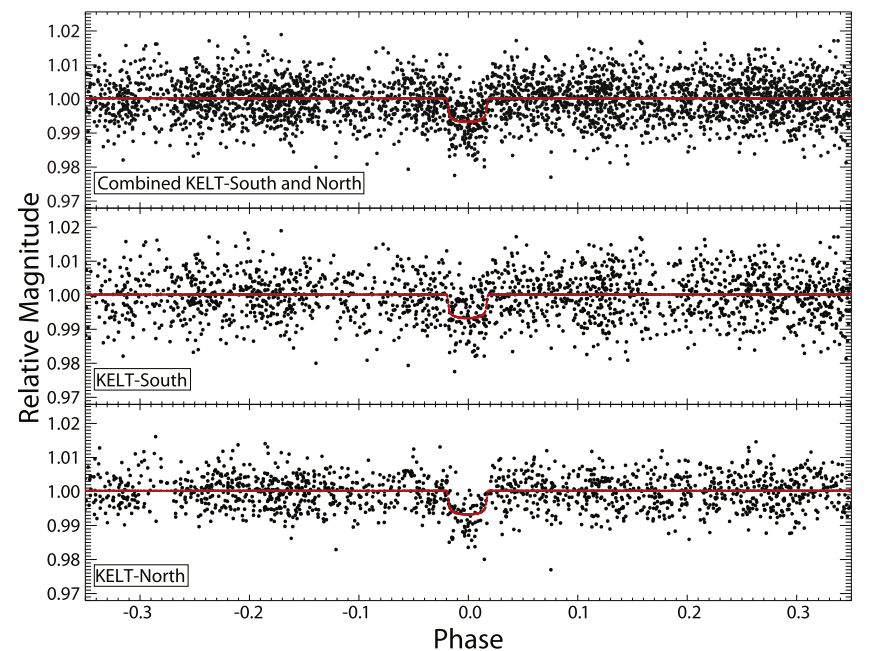

Figure 1. Combined KELT-South and KELT-North light curve (top), the KELT-South light curve alone (middle), and the KELT-North discovery light curve (bottom) for KELT-19Ab. Each has been phase folded to the discovery period of 4.6117449 days. The red line corresponds to an EXOFAST model of the combined light curve. (Supplemental data for this figure are available in the online journal.) The data used to create this figure are available.

Virtually all slower rotators (including KELT-19A) are measured to be chemically peculiar, although there may well be exceptions. For example, a very young slow rotator might not yet have had time to develop unusual abundance patterns. Empirically, most slowly rotating Am stars are also in binaries (Abt \& Levy 1985), as is the case for KELT-19A (see Sections 2.3 and 2.4.3). This may be due to tidal braking of the A star, although in the case of KELT-19, the stellar companion is too distant for such tidal braking to be effective.

\section{Discovery and Follow-up Observations}

We provide a brief summary of the KELT survey data reduction process and present the results in Section 2.1. Section 2.2 presents our ground-based time-series follow-up photometric observations, Section 2.3 presents our highcontrast adaptive optics imaging, and Section 2.4 presents our spectroscopic follow-up observations.

\subsection{KELT Observations and Photometry}

KELT-19Ab is located in a field that is monitored by both KELT telescopes, centered on $\alpha=07^{\mathrm{h}} 39^{\mathrm{m}} 36^{\mathrm{s}}, \delta=+03^{\circ} 00^{\prime}$ $00^{\prime \prime}$ (J2000). This field is labeled internally as KELT-South field 06 (KS06) and KELT-North field 14 (KN14). The reduction and candidate selection process for KELT-South and KELT-North are described in detail in Kuhn et al. (2016) and Siverd et al. (2012), respectively. From our analysis of 2636 images from KS06 (UT 2010 March 02 to 2013 May 10) and 2092 images from KN14 (UT 2011 October 11 to UT 2013 March 26), KJ06C009789 (KELT-19Ab) was identified as a top candidate. Figure 1 shows the combined KELT-South and KELT-North light curve (top), the KELT-South light curve alone (middle), and the KELT-North light curve (bottom) for KELT-19Ab. KELT-19 (BD+07 1721) is located at $\alpha=07^{\mathrm{h}} 26^{\mathrm{m}} 02^{\mathrm{s}} .2895, \quad \delta=+07^{\circ} 36^{\prime} 56$ !" $^{\prime \prime} 34$ (J2000). This is the second planet discovered through a combination of

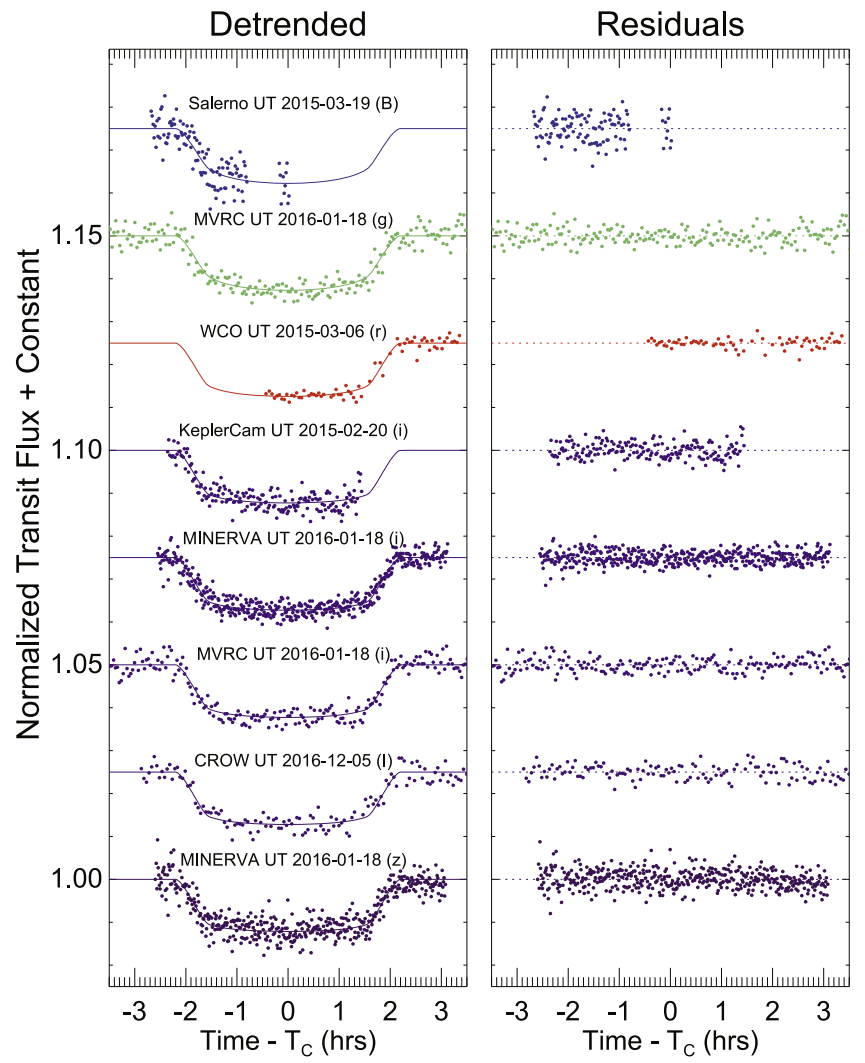

Figure 2. Follow-up transit photometry of KELT-19. Left panel: detrended transit light curves arbitrarily shifted on the vertical axis for clarity. The overplotted solid lines are the best-fit transit model from the adopted global fit documented in Table 5. Right panel: transit model residuals. The labels are as follows: $\quad$ Salerno $=$ Salerno University Observatory $0.35 \mathrm{~m}$ telescope; MVRC = Manner-Vanderbilt $0.6 \mathrm{~m}$ RCOS Telescope; WCO = Westminster College Observatory $0.35 \mathrm{~m}$ telescope; KeplerCam $=1.2 \mathrm{~m}$ telescope at FLWO; MINERVA = MINiature Exoplanet Radial Velocity Array of $0.7 \mathrm{~m}$ telescopes; CROW = Canela's Robotic Observatory 0.3 m LX200 Telescope. (Supplemental data for this figure are available in the online journal.) The data used to create this figure are available.

KELT-South and KELT-North observations, KELT-17b being the first (Zhou et al. 2016a).

\subsection{Photometric Time-series Follow-up}

The KELT collaboration includes a world-wide team of ground-based follow-up observers known as the KELT FollowUp Network (KELT-FUN). KELT-FUN currently includes members from $\approx 60$ institutions. The KELT-FUN team acquired follow-up time-series photometry of KELT-19Ab transits to better determine the system parameters and to check for transit false positives. We used the Tapir software package (Jensen 2013) to schedule follow-up observations. We obtained six full and three partial transits in multiple bandpasses from $g$ to $z$ between 2015 February and 2016 December. Figure 2 shows all the transit follow-up light curves assembled. A summary of the follow-up photometric observations is shown in Table 1 . We find consistent $R_{\mathrm{P}} / R_{\star}$ ratios in all light curves across the optical bands, helping to rule out false positives due to blended eclipsing binaries. Figure 3 shows all transit follow-up light curves from Figure 2 
Table 1

Summary of Photometric Observations

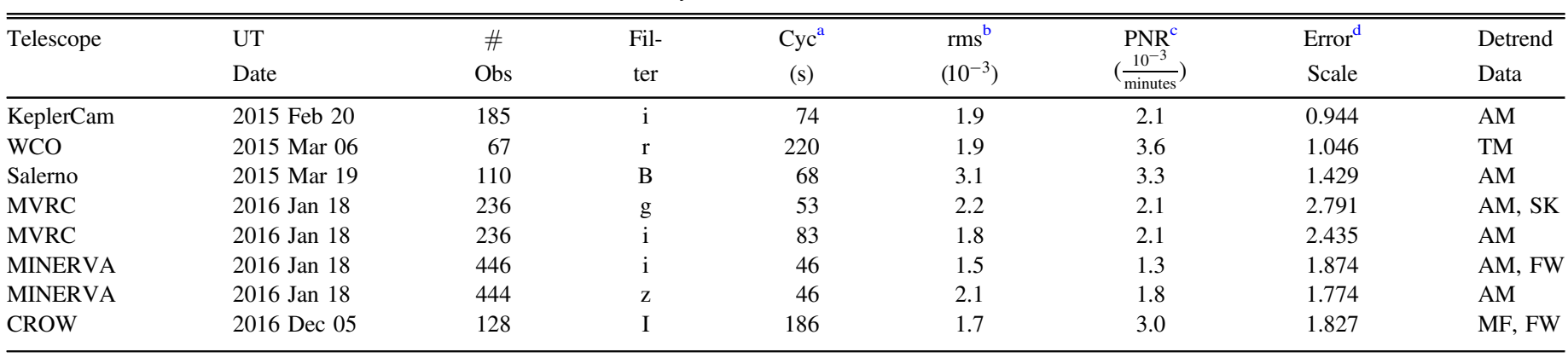

Notes. See Figure 2 for a description of the telescope naming convention; $\mathrm{AM}=$ airmass; $\mathrm{TM}=$ time; $\mathrm{SK}=$ sky background; FW $=$ average FWHM in image; $\mathrm{MF}=$ baseline offset at meridian flip.

${ }^{\text {a }}$ Cycle time in seconds, calculated as the mean of exposure time plus dead time during periods of back-to-back exposures.

${ }^{b} \mathrm{rms}$ of residuals from the best-fit model in units of $10^{-3}$.

${ }^{c}$ Photometric noise rate in units of $10^{-3}$ minute ${ }^{-1}$, calculated as $\mathrm{rms} / \sqrt{\Gamma}$, where rms is the scatter in the light-curve residuals and $\Gamma$ is the mean number of cycles (exposure time and dead time) per minute during periods of back-to-back exposures (adapted from Fulton et al. 2011).

${ }^{\mathrm{d}}$ Error scaling factor determined by MULTIFAST (see Section 4.4).

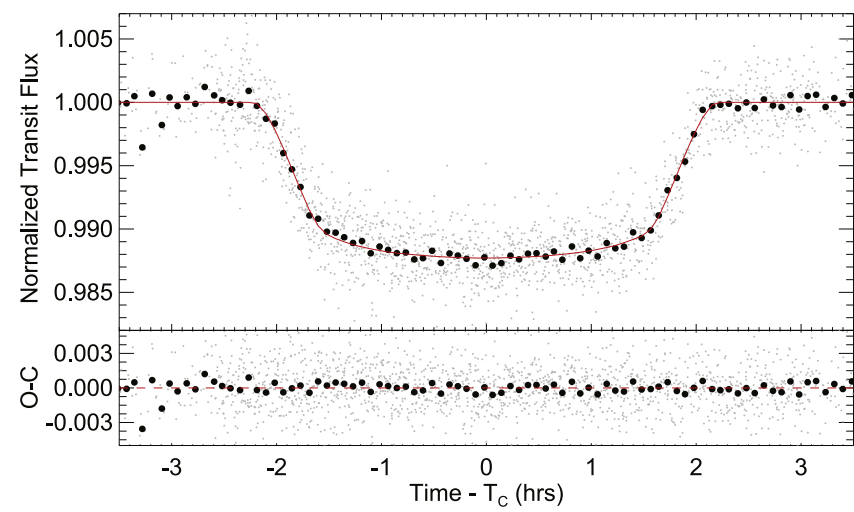

Figure 3. All detrended follow-up transits combined (small light-gray points) and binned in five-minute intervals (large black points) and overplotted with the best-fit global model (solid red line). The model shows the average limb darkening weighted by the number of transits in each band. The model residuals are shown in the bottom panel. The binned light curve data are not used in the analysis and are presented here to illustrate the overall statistical power of the follow-up photometry.

combined and binned in five-minute intervals. This combined and binned light curve is not used for analysis, but rather to show the best combined behavior of the transit.

All photometric follow-up observations were reduced with the AstroImage J (AIJ) software package ${ }^{40}$ (Collins et al. 2017). We were careful to ensure that all observatory computers were referenced either through a network connection to a stratum 1 timing source or to a GPS stratum 1 timing source, and that all quoted mid-exposure times were properly reported in barycentric Julian dates at mid-exposure (BJD ${ }_{\mathrm{TDB}}$; Eastman et al. 2010).

\subsubsection{KeplerCam}

We observed an $i$-band transit ingress from KeplerCam on the $1.2 \mathrm{~m}$ telescope at the Fred Lawrence Whipple Observatory (FLWO) on UT 2015 February 20. KeplerCam has a single $4 \mathrm{~K} \times 4 \mathrm{~K}$ Fairchild CCD 486 with an image scale of 0 "' 366 pixel $^{-1}$ and a field of view of 23 ! $1 \times 23$ ! 1 .

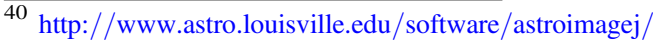

\subsection{2. $\mathrm{WCO}$}

We observed an $r$-band transit egress from the Westminster College Observatory (WCO) on UT 2015 March 06. The observations were conducted from a $0.35 \mathrm{~m} f / 11$ Celestron C14 Schmidt-Cassegrain telescope equipped with an SBIG STL$6303 \mathrm{E} C \mathrm{CD}$ with a $3 \mathrm{~K} \times 2 \mathrm{~K}$ array of $9 \mu \mathrm{m}$ pixels. The resulting images have a $24^{\prime} \times 16^{\prime}$ field of view and $11^{\prime \prime} 4$ pixel $^{-1}$ image scale at $3 \times 3$ pixel binning.

\subsubsection{Salerno}

We observed an $R$-band transit ingress on UT 2015 March 19 from the Salerno University Observatory in Fisciano Salerno, Italy. The observing setup consists of a $0.35 \mathrm{~m}$ Celestron C14 SCT and an SBIG ST2000XM $1600 \times 1200$ $\mathrm{CCD}$, yielding an image scale of $0 . " 54 \mathrm{pixel}^{-1}$.

\subsubsection{MINERVA}

We observed a full transit simultaneously in the Sloan $r$-, $i$-, and $z$-bands using three of the MINERVA Project telescopes (Swift et al. 2015) on the night of UT 2016 January 18. MINERVA uses four $0.7 \mathrm{~m}$ PlaneWave CDK-700 telescopes that are located on Mt. Hopkins, AZ, at FLWO. While the four telescopes are normally used to feed a single spectrograph, we used three MINERVA telescopes in their photometric imaging mode for the KELT-19 observations. The telescopes were equipped with Andor iKON-L $2048 \times 2048$ cameras, which gave a field of view of $20.9 \times 20^{\prime} 9$ and a plate scale of $0{ }^{\prime \prime} 6 \mathrm{pixel}^{-1}$. The MINERVA telescope conducting the $r$-band observations experienced a 2!.6 tracking jump during the time of egress. The resulting change in the position of the field on the detector produces a relatively large change in the baseline level of the light curve just after the beginning of egress. Furthermore, because of imperfect flat-field images, the baseline offset differs by $\sim \pm 1$ percent depending on the set of comparison stars selected. The different baseline offsets produce transit center times that differ by $\sim \pm 8$ minutes, even with detrending parameters included in the model to attempt to compensate for the baseline offset. Because of the unreliable detrending results and because we simultaneously observed four additional light curves on UT 2016 January 18, the $r$-band 
light curve is not included in the analysis to avoid the potential of improperly biasing the linear ephemeris derived from our global modeling effort (see Section 4.4.5).

\subsubsection{MVRC}

We observed a full transit from the Manner-Vanderbilt Ritchey-Chrétien (MVRC) telescope located at the Mt. Lemmon summit of Steward Observatory, AZ, on UT 2016 January 18. Exposures were taken in alternating $g$ - and $i$-band filters, yielding pseudo-simultaneous observations in the two filters. The observations employed a $0.6 \mathrm{~m} / 8 \mathrm{RC}$ Optical Systems Ritchey-Chrétien telescope and an SBIG STX-16803 CCD with a $4 \mathrm{~K} \times 4 \mathrm{~K}$ array of $9 \mu \mathrm{m}$ pixels, yielding a $26 ! 6 \times 26^{\prime} \cdot 6$ field of view and 0!' 39 pixel $^{-1}$ image scale.

\subsection{6. $C R O W$}

We observed a full I-band transit from Canelas Robotic Observatory (CROW) in Portalegre, Portugal on UT 2016 December 05 . The observatory is equipped with a $0.3 \mathrm{~m}$ Schmidt-Cassegrain telescope and a KAF-3200E CCD, having a $30^{\prime} \times 20^{\prime}$ field of view and a pixel scale of 0 "' 84 pixel $^{-1}$.

\subsection{High-Contrast Imaging}

KELT-19 was observed on the night of UT 2016 December 18 at Palomar Observatory with the 200" Hale Telescope using the near-infrared adaptive optics (AO) system P3K and the infrared camera PHARO (Hayward et al. 2001). PHARO has a pixel scale of 0.1025 pixel $^{-1}$ and a full field of view of approximately $25^{\prime \prime}$. The data were obtained with a narrow-band $\operatorname{Br}-\gamma$ filter $\left(\lambda_{o}=2.18 ; \Delta \lambda=0.03 \mu \mathrm{m}\right)$ and a standard $J$-band filter $\left(\lambda_{o}=1.246 ; \Delta \lambda=0.162 \mu \mathrm{m}\right)$.

The AO data were obtained in a five-point quincunx dither pattern with each dither position separated by $4^{\prime \prime}$. Each dither position is observed three times, each offset from the previous image by $0 . " 5$ for a total of 15 frames; the integration time per frame was $17 \mathrm{~s}$ in both the $\mathrm{Br}-\gamma$ and $J$ filters. We use the dithered images to remove sky background and dark current, and then align, flat-field, and stack the individual images. The PHARO AO data have a resolution of 0!" 11 and 0". 25 (FWHM) in the $\mathrm{Br}-\gamma$ and $J$ filters, respectively.

The sensitivities of the final combined AO image were determined by injecting simulated sources azimuthally around KELT-19A every $45^{\circ}$ at separations of integer multiples of the central source's FWHM (Furlan et al. 2017). The brightness of each injected source was scaled until standard aperture photometry detected it with $5 \sigma$ significance. The resulting brightness of the injected sources relative to KELT-19A set the contrast limits at that injection location. The $5 \sigma$ limit at each separation was determined from the average of all of the determined limits at that separation. The contrast sensitivity curve shown in Figure 4 represents the $5 \sigma$ limits of the imaging data in $\Delta$ magnitude versus angular separation in arcseconds. The slight decrease in sensitivity near $1^{\prime \prime}$ is caused by an increase in the relative brightness of the diffraction spikes in comparison to the smoothly declining point-spread function of the target.

For KELT-19, a nearby stellar companion was detected in both the $\mathrm{Br}-\gamma$ and $J$ filters. The presence of the blended companion must be taken into account to obtain the correct transit depth and planetary radius (e.g., Ciardi et al. 2015). The companion separation was measured from the $\mathrm{Br}-\gamma$ image and

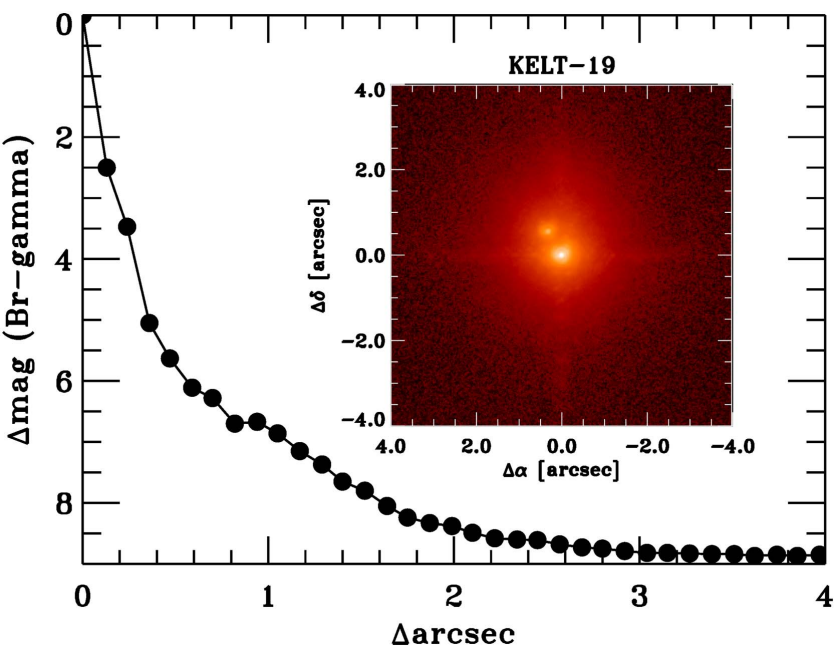

Figure 4. Contrast sensitivity and inset image of KELT-19 in Br- $\gamma$ as observed with the Palomar Observatory Hale Telescope adaptive optics system; the secondary companion is clearly detected. The $5 \sigma$ contrast limit in $\Delta$ magnitude is plotted against angular separation in arcseconds. The slight decrease in sensitivity near $1^{\prime \prime}$ is caused by an increase in the relative brightness of the diffraction spikes in comparison to the smoothly declining point-spread function of the target.

found to be $\Delta \alpha=0$ "' $32 \pm 0$ ". 02 and $\Delta \delta=0$ ". $55 \pm 0 . " 02$, which is a projected separation of 0 ". $64 \pm 0$ ". 03 at a position angle of $30.2 \pm 2.5$ degrees. At a distance of $255 \pm 15 \mathrm{pc}$ (see Section 4.1), the companion has a projected separation from the primary star of $\approx 160$ au. The positional offset uncertainties between the two stars are based upon the uncertainties in the positional fit to the centroids of the point-spread functions of the stars and is approximately 0.1 of a pixel, corresponding to 2.5 mas. No distortion map was applied to the images; however, the optical distortion is $0.4 \%$ or less in the narrow camera mode for PHARO (Hayward et al. 2001).

The stars have blended 2MASS magnitudes of $J=$ $9.343 \pm 0.026 \mathrm{mag}$ and $K_{s}=9.196 \pm 0.023 \mathrm{mag}$. The stars have measured magnitude differences of $\Delta J=2.50 \pm 0.06 \mathrm{mag}$ and $\Delta K_{s}=2.045 \pm 0.03 \mathrm{mag}$; the $J$-band differential measurement is less certain because of the poor $\mathrm{AO}$ correction in that filter on the night of the observations. $\mathrm{Br}-\gamma$ has a central wavelength that is sufficiently close to $K s$ to enable the deblending of the 2MASS magnitudes into the two components. The primary star has deblended (real) apparent magnitudes of $J_{1}=9.45 \pm 0.03$ mag and $K s_{1}=9.35 \pm 0.02 \mathrm{mag}$, corresponding to a color of $\left(J-K_{s}\right)_{1}=0.10 \pm 0.04 \mathrm{mag} ;$ the companion star has deblended (real) apparent magnitudes of $J_{2}=11.95 \pm 0.06$ mag and $K s_{2}=11.40 \pm 0.03 \mathrm{mag}$, corresponding to a color $\left(J-K_{s}\right)_{2}=0.55 \pm 0.07 \mathrm{mag}$. The uncertainties in the stellar colors are dominated by the uncertainty in the $J$-band measurement. Using the Casagrande et al. (2010) relations, the colors give $T_{\text {eff }}=7190_{-250}^{+270} \mathrm{~K}$ for the primary and $T_{\text {eff }}=5030_{-240}^{+260} \mathrm{~K}$ for the companion, which are consistent with the effective temperatures derived from the spectral energy distribution (SED) analysis in Section 4.1 and the spectral analysis in Section 2.4.5.

\subsection{Spectroscopic Follow-up}

\subsubsection{TRES at FLWO}

To constrain the planet mass and enable eventual Doppler tomographic (DT) detection of KELT-19Ab, we obtained a total of 60 spectroscopic observations of the host star with the 


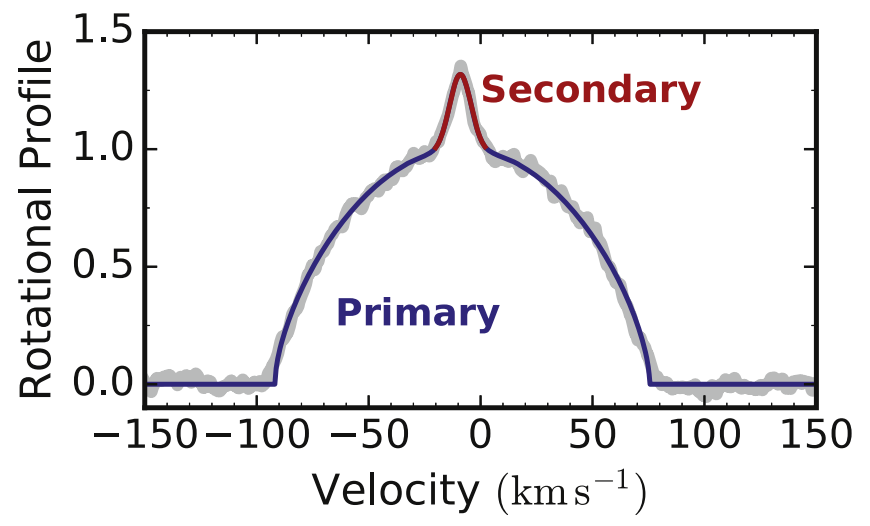

Figure 5. Example broadening kernel of KELT-19 (gray line), as observed by TRES, showing the spectroscopic binary nature of the system. We fit both spectroscopic components simultaneously to obtain the radial velocities of both stellar components; the best-fit profiles for the primary and companion are shown in blue and red, respectively.

Tillinghast Reflector Echelle Spectrograph (TRES) on the $1.5 \mathrm{~m}$ telescope at the Fred Lawrence Whipple Observatory, Arizona, USA. Each spectrum delivered by TRES has a spectral resolution of $\lambda / \Delta \lambda=44,000$ over the wavelength range of $3900-9100 \AA$ in 51 échelle orders. A total of seven observations were obtained during the out-of-transit portions of the planet's orbit to constrain its mass (Section 2.4.3). Two spectroscopic transits were observed, on 2016 February 24 and 2016 November 08, for the Doppler tomographic analysis. The observations on 2016 February 24 were plagued by bad weather, and were discarded. The transit sequence obtained on 2016 November 08, totaling 24 spectra, successfully revealed the planetary transit, and were used in the analysis described in Section 2.4.4.

\subsubsection{HJST at McDonald}

To provide additional constraints on the planet mass, we obtained 14 spectra of KELT-19 covering the entire orbital phase with the $2.7 \mathrm{~m}$ Harlan J. Smith Telescope (HJST) at McDonald Observatory and the Robert G. Tull Coudé spectrograph (Tull et al. 1995) in its TS23 configuration. This is a cross-dispersed échelle spectrograph with a resolving power of $R=60,000$ and coverage from 3570 to $10,200 \AA$ (complete below $5691 \AA$ ) over 58 orders. The first 2 spectra (from 2016 October) have exposure lengths of $\sim 375 \mathrm{~s}$, while the last 12 (from 2016 December) have $1200 \mathrm{~s}$ exposure lengths.

\subsubsection{Radial Velocities}

The nearby stellar companion (see Section 2.3) is blended with the primary in our spectroscopic observations. Because of the resulting composite spectra, our radial velocity analysis is somewhat modified from previous KELT papers. For each observation, we derived a line broadening kernel via a leastsquares deconvolution (following Donati et al. 1997; Collier Cameron et al. 2010), from which both spectroscopic components can be identified (Figure 5). To derive radial velocities and rotational broadening parameters, we fit for the two spectroscopic components simultaneously across all available out-of-transit spectra, allowing for independent radial velocities of the two components, while requiring all observations to have the same velocity broadening parameters. The
TRES and HJST observations were fit independently, since they are subjected to different instrumental broadening, and the broadening profiles were derived from different spectral wavelength regions.

From the simultaneous fit, we find that the out-of-transit broadening profile can best be described by a rapidly rotating primary star and a faint, slowly rotating secondary star. The primary component has a rotational broadening velocity of $v \sin I_{*}=84.1 \pm 2.1 \mathrm{~km} \mathrm{~s}^{-1}$ and a combined macro- and microturbulent broadening of $3.4 \pm 2.0 \mathrm{~km} \mathrm{~s}^{-1}$. The effect of instrumental broadening is taken into account separately in the global modeling. The secondary component has a line broadening velocity of $8.23 \pm 0.11 \mathrm{~km} \mathrm{~s}^{-1}$, which includes the influence of instrumental $\left(6.8 \mathrm{~km} \mathrm{~s}^{-1}\right)$, rotational, and turbulent sources. We find a flux ratio of $F_{B} / F_{A}=0.0270 \pm 0.0034$ to the total light of the system over the wavelength range $5200 \pm 150 \AA$. This flux ratio is consistent with the AO observations of the spatially separated companion, and with the interpretation that the secondary companion is a G-dwarf associated with the system (Section 4.1).

We estimate the absolute center of mass radial velocity for KELT-19 from the Mg b region of our TRES spectra. We examined the mean of (1) all velocities, (2) the out-of-transit velocities, and (3) the high signal-to-noise ratio $(\mathrm{S} / \mathrm{N})$ velocities and concluded that the best nominal value and uncertainty representing the absolute radial velocity (RV) of the KELT-19 system is $-7.9 \pm 0.5 \mathrm{~km} \mathrm{~s}^{-1}$. The absolute RV was then adjusted to the International Astronomical Union (IAU) Radial Velocity Standard Star system via a correction of $-0.62 \mathrm{~km} \mathrm{~s}^{-1}$ resulting in a final value of $\mathrm{RV}_{\mathrm{IAU}}=-8.5 \pm 0.5 \mathrm{~km} \mathrm{~s}^{-1}$. The correction primarily adjusts for the gravitational redshift, which is not included in the library of synthetic template spectra.

The TRES and HJST out-of-transit velocities are shown in Figure 6, and presented in Table 2. As discussed in Section 4.4, the RV semi-amplitude of the primary can be constrained to be $<0.35 \mathrm{~km} \mathrm{~s}^{-1}$, confirming that the transiting companion is of planetary mass. We also confirmed that the velocity of the stellar companion does not vary, and it is constrained to be $<2.31 \mathrm{~km} \mathrm{~s}^{-1}$ at $1 \sigma$, and $<7.50 \mathrm{~km} \mathrm{~s}^{-1}$ at $3 \sigma$. We note that the greatest hurdle to obtaining precise radial velocities for KELT$19 \mathrm{~A}$ is its rapid rotational velocity. In comparison, we often reach $\sim 10 \mathrm{~m} \mathrm{~s}^{-1}$ precision for slowly rotating non-active stars with TRES (Quinn et al. 2014). The systemic velocity of the primary $\left(-8.5 \pm 0.5 \mathrm{~km} \mathrm{~s}^{-1}\right)$ is consistent with that of the companion $\left(-9.4 \pm 1.0 \mathrm{~km} \mathrm{~s}^{-1}\right)$, which we interpret as KELT19A and KELT-19B being bound. Assuming a $0.5 M_{\odot}$ bound companion in a circular, nearly edge-on orbit with radius $160 \mathrm{au}, \mathrm{KELT}-19 \mathrm{~B}$ would cause a maximum acceleration of KELT-19A (at conjunction or opposition) of $\sim 4 \mathrm{~m} \mathrm{~s}^{-1} \mathrm{yr}^{-1}$. Given the current relatively low RV precision due to the rapid rotation of the primary, it is not surprising that an RV trend is not detected in the current data, and furthermore would not be detected for the foreseeable future. However, under the same assumptions, KELT-19A would cause a maximum acceleration of KELT-19B of $\sim 12 \mathrm{~m} \mathrm{~s}^{-1} \mathrm{yr}^{-1}$, which might be detectable after several years with RV instruments that can achieve precisions of a few $\mathrm{m} \mathrm{s}^{-1}$ for a $J=12 \mathrm{mag}$ star, given the relatively low $v \sin I_{*}$ of $\sim 4 \mathrm{~km} \mathrm{~s}^{-1}$ of the secondary. 


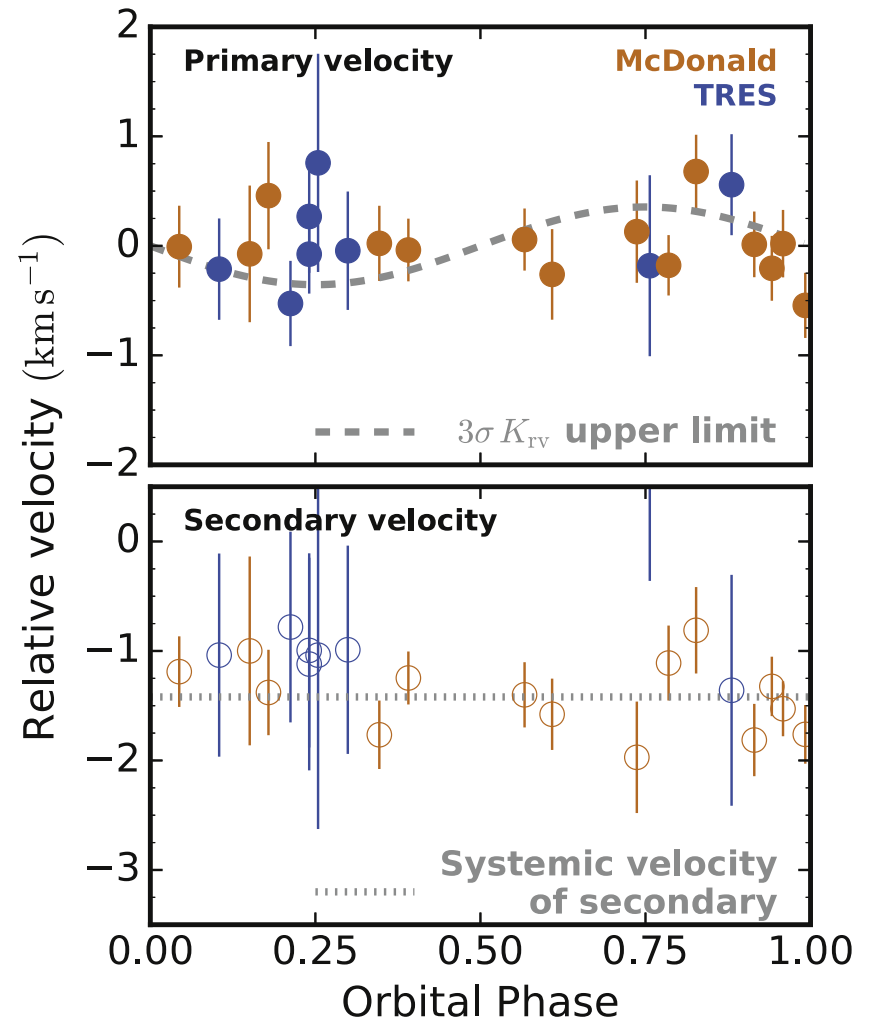

Figure 6. Radial velocities of the two stellar components in the KELT-19 system, phase folded to the transit period. We can place a $3 \sigma$ upper limit on the RV semi-amplitude $K$ of $0.35 \mathrm{~km} \mathrm{~s}^{-1}$, confirming that the transiting companion is of planetary mass. The velocity of the stellar companion is constrained to be $<2.31 \mathrm{~km} \mathrm{~s}^{-1}$ at $1 \sigma$, and $<7.50 \mathrm{~km} \mathrm{~s}^{-1}$ at $3 \sigma$. The systemic velocity of the companion is similar to that of the primary, consistent with the interpretation that they are physically associated. The primary velocities are plotted in the top panel, and secondary velocities in the bottom panel. The systemic velocity of the primary has been subtracted for all measurements. The TRES velocities are plotted in blue, and McDonald velocities in orange.

\subsubsection{Doppler Tomographic Observations}

As a star rotates, one hemisphere moves toward the observer relative to the integrated stellar RV, which produces light with a blueshifted spectrum. The other hemisphere moves away from the observer, producing light with a redshifted spectrum. In total, this produces rotationally broadened spectral lines. As a planet transits a star, differing blue- and/or redshifted stellar spectral components are obscured by the shadow of the planet on the star. The planet shadow thus produces a spectral line profile distortion that varies in velocity space (except for the case of a polar orbit) as the transit progresses from ingress to egress. The measurement of the motion of the distortion can be modeled to reveal the system's spin-orbit misalignment, $\lambda$, and the impact parameter, $b$, of the planet's orbit relative to the stellar disk. See Johnson et al. (2014) for a more technical description.

To confirm that a transiting companion is indeed orbiting the primary star in the KELT-19 system, and to measure the projected spin-orbit angle and impact parameter of the planetary orbit, we performed a Doppler tomographic analysis of the spectroscopic transit observed by TRES on 2016 November 08. Line broadening profiles were derived for each observation via a least-squares deconvolution analysis (following Donati et al. 1997; Collier Cameron et al. 2010; Zhou et al. 2016a). A master broadening profile was calculated by
Table 2

Radial Velocity Measurements of KELT-19

\begin{tabular}{|c|c|c|c|c|}
\hline $\mathrm{BJD}_{\mathrm{TDB}}$ & $\begin{array}{c}\text { Primary } \\
\text { RV } \\
\left(\mathrm{m} \mathrm{s}^{-1}\right)\end{array}$ & $\begin{array}{c}\text { Primary } \\
\sigma_{\mathrm{RV}}^{\mathrm{a}} \\
\left(\mathrm{m} \mathrm{s}^{-1}\right)\end{array}$ & $\begin{array}{c}\text { Secondary } \\
\text { RV } \\
\left(\mathrm{m} \mathrm{s}^{-1}\right)\end{array}$ & $\begin{array}{c}\text { Secondary } \\
\sigma_{\mathrm{RV}^{\mathrm{a}}} \\
\left(\mathrm{m} \mathrm{s}^{-1}\right)\end{array}$ \\
\hline \multicolumn{5}{|l|}{ TRES } \\
\hline 2457118.717801 & -8322 & 677 & -6315 & 2185 \\
\hline 2457323.926960 & -7382 & 817 & -9178 & 1588 \\
\hline 2457704.974522 & -7582 & 378 & -9499 & 1054 \\
\hline 2457706.006779 & -8353 & 379 & -9178 & 928 \\
\hline 2457706.905449 & -8185 & 443 & -9129 & 951 \\
\hline 2457715.859122 & -7872 & 422 & -9260 & 971 \\
\hline 2457761.845292 & -8667 & 319 & -8922 & 871 \\
\hline \multicolumn{5}{|l|}{ McDonald } \\
\hline 2457685.865924 & -7126 & 413 & -9227 & 509 \\
\hline 2457687.904483 & -6797 & 434 & -8635 & 391 \\
\hline 2457732.803281 & -7243 & 266 & -9069 & 330 \\
\hline 2457732.925982 & -7461 & 262 & -8580 & 271 \\
\hline 2457733.004039 & -7236 & 273 & -8784 & 251 \\
\hline 2457733.890461 & -7329 & 552 & -8256 & 862 \\
\hline 2457734.795794 & -7234 & 304 & -9021 & 312 \\
\hline 2457734.998676 & -7294 & 253 & -8503 & 241 \\
\hline 2457735.810347 & -7199 & 251 & -8656 & 298 \\
\hline 2457736.002794 & -7517 & 366 & -8834 & 326 \\
\hline 2457736.816475 & -7434 & 244 & -8366 & 342 \\
\hline 2457737.008672 & -6577 & 297 & -8067 & 395 \\
\hline 2457737.771236 & -7800 & 263 & -9017 & 268 \\
\hline 2457738.009789 & -7263 & 331 & -8445 & 322 \\
\hline
\end{tabular}

Note. Because of the rapidly rotating host star, we were unable to derive bisector spans.

${ }^{\mathrm{a}} \mathrm{RV}$ errors before being scaled by MULTIFAST.

combining the out-of-transit profiles. Each observation was then subtracted from the master broadening profile, revealing the spectroscopic shadow of the transiting planet, as shown in Figure 7. The Doppler tomographic signal was modeled as per Gaudi et al. (2017). Limb-darkening parameters were adopted from Claret (2004) for the photometric $V$ band, similar to the wavelength region from which the broadening profiles were derived.

\subsubsection{Stellar Parameters from Spectra}

Because the spectrum of KELT-19A includes the light from KELT-19B (see Sections 2.4.3 and 2.3), standard spectral synthesis or fitting techniques that ignore the influence of the secondary on the primary line profiles may be susceptible to systematic bias. We therefore applied a two-dimensional crosscorrelation analysis (TODCOR; Zucker \& Mazeh 1994) using pairs of synthetic spectra to identify the stellar parameters that provided the best fit to the observed composite spectra. For this analysis, we used the TRES spectra of KELT-19 and the CfA library of synthetic spectra, which were generated by John Laird using Kurucz model atmospheres (Kurucz 1992) and a linelist compiled by Jon Morse. The synthetic grid covers the wavelength range 5050-5350 $\mathrm{A}$, and has spacing of $250 \mathrm{~K}$ in $T_{\text {eff }}$ and 0.5 dex spacing in $\log g_{\star}$ and $[\mathrm{m} / \mathrm{H}]$. We note that this latter parameter is a scaled solar bulk metallicity, rather than the iron abundance, $[\mathrm{Fe} / \mathrm{H}]$. It is generally a reasonable assumption that the two quantities are similar, but it might not be the case for stars exhibiting peculiar abundances (like many A stars). Throughout the paper, we do use $[\mathrm{m} / \mathrm{H}]$ and $[\mathrm{Fe} / \mathrm{H}]$ interchangeably, but because we neither derive nor impose 


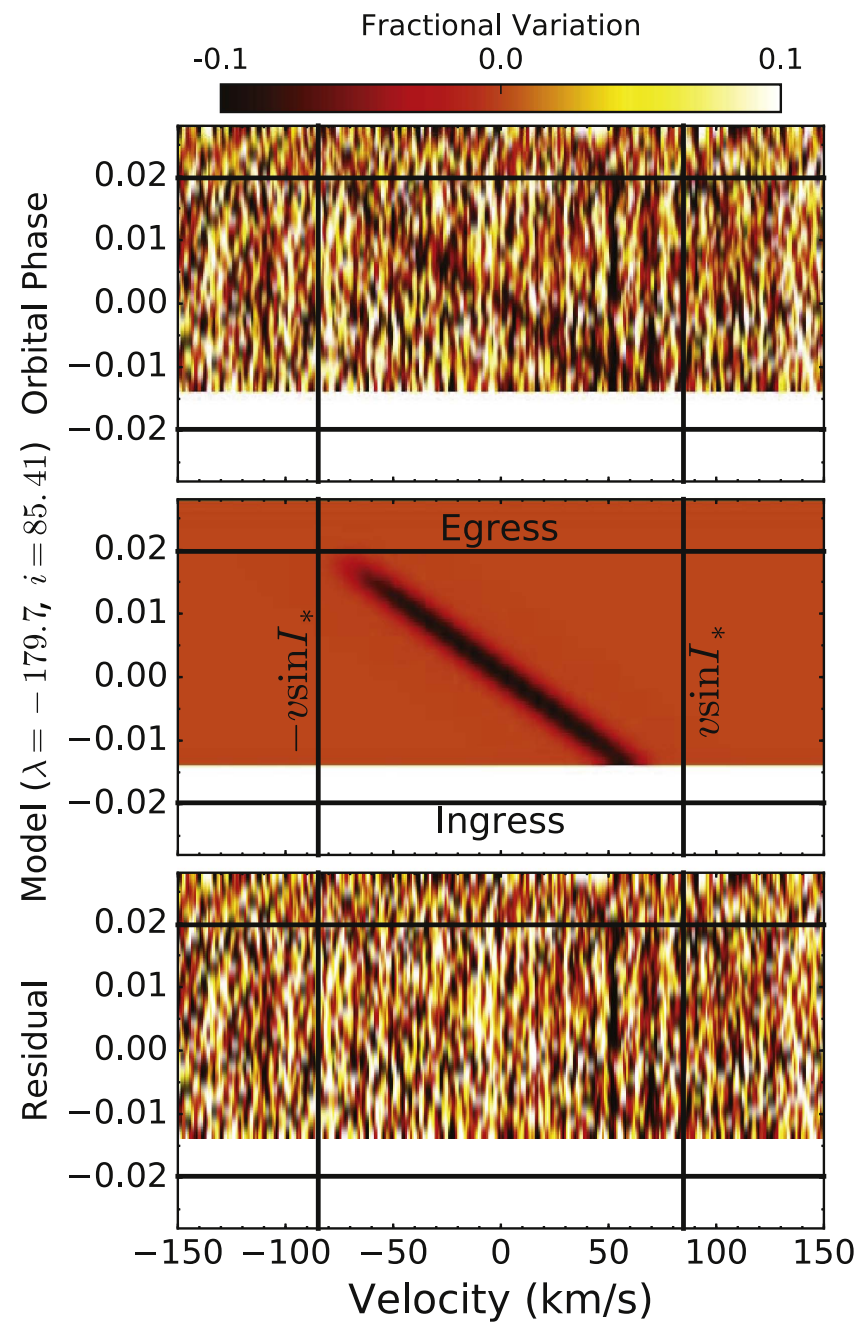

Figure 7. Doppler tomographic line profile plot. The top panel shows the spectroscopic data, the middle panel shows the derived model, and the bottom panel shows the residuals. In each panel, the vertical lines denote the width of the convolution kernel (i.e., $\pm v \sin I_{*}$ ), and the horizontal lines show the duration of the transit. Time increases from bottom to top. Each color-scale row indicates the deviation of the line profile at that time from the out-of-transit line profile, with dark regions of the plot indicating regions of the in-transit line profile that are shallower with respect to the out-of-transit line profile. The Doppler tomographic signal implies a retrograde orbit for the planet, as the line profile perturbation moves from the red wing of the line profile across to the blue wing. The planet moves in a corresponding manner during the transit, from obscuring the redshifted hemisphere of the star across to the blueshifted hemisphere.

strong constraints on the metallicity, we expect that any differences between the two quantities will have negligible effects on our results.

We ran TODCOR on all combinations of templates in the (6D) parameter space spanning temperatures $6000 \leqslant$ $T_{\text {eff, }} \leqslant 8500 \mathrm{~K}$ and $3750 \leqslant T_{\text {eff, }} \leqslant 6750 \mathrm{~K}$, surface gravities $3.0 \leqslant \log g_{\star} \leqslant 5.0$, and metallicities $-1.5 \mathrm{dex} \leqslant[\mathrm{m} / \mathrm{H}] \leqslant$ +0.5 dex for both stars. We allowed the primary and secondary metallicities to be fit independently because even if the two stars formed together, many A stars display peculiar photospheric metallicities. The mean TODCOR correlation coefficient from each of these $\sim 37,000$ template pairs defines a $6 \mathrm{D}$ surface (the axes corresponding to the six stellar parameters), on which we interpolate to the peak and adopt the corresponding stellar parameters. The result comes with several caveats. Derived spectroscopic stellar parameters are highly covariant-temperatures, metallicities, and gravities can be altered simultaneously to obtain very similar spectra over relatively large ranges of parameter space-so this degeneracy must be broken with independent constraints. In our case, we have derived the primary surface gravity $\left(\log g_{\star}=4.127\right)$ from constraints on the stellar density, mass, and radius as part of the global system fit (see Section 4.4). Because $\log g_{\star}$ is determined so precisely, even a $3 \sigma$ error in this value has minimal effect on the other parameters. As a result, we fixed it in the TODCOR analysis for simplicity. Additionally, the secondary spectrum possesses a very low signal-to-noise ratio, so its parameters are poorly constrained by the spectra alone. Instead, we required it to be a main-sequence companion $\log g_{\star} \sim 4.5$, with a temperature of $5200 \mathrm{~K}$ (as derived in our initial SED analysis; Section 4.1). We note that the projected rotational velocities, $v \sin I_{*}$, are nearly orthogonal to the other parameters, so we fixed these to simplify the analysis and reduce computation time: the primary $v \sin I_{*}$ was set to $84.1 \mathrm{~km} \mathrm{~s}^{-1}$ (see Section 2.4.3), while the secondary $v \sin I_{*}$ was estimated to be $\sim 2 \mathrm{~km} \mathrm{~s}^{-1}$ via an empirical gyrochronology relation (Mamajek \& Hillenbrand 2008) and the age and colors derived from the initial SED and isochrone analysis.

Under these constraints, we find the following parameters: $T_{\text {eff,A }}=7505 \pm 104 \mathrm{~K} ;[\mathrm{m} / \mathrm{H}]_{A}=+0.24 \pm 0.16 ;[\mathrm{m} / \mathrm{H}]_{B}=$ $-0.26 \pm 0.35$. The reported errors include contributions from both formal and correlated errors. It is interesting to note that the primary metallicity is 0.5 dex higher than that of the secondary, albeit at low confidence because of the noisy secondary spectrum. We would expect to observe this difference if the primary is an Am star, a possibility we explore in Section 2.4.6. Given the uncertainty in the metallicities - and the possibility that the photospheric spectrum of the primary is not representative of its true metallicity -we choose to adopt a broad metallicity prior appropriate for the solar neighborhood $([\mathrm{Fe} / \mathrm{H}]=0.0 \pm 0.5 \mathrm{dex})$ in our subsequent global modeling. The main result of the TODCOR analysis, then, is a spectroscopic temperature for the primary of $T_{\text {eff }}=7505 \pm 104 \mathrm{~K}$.

\subsubsection{KELT-19A is Likely an Am Star}

As noted in the introduction, KELT-19A has a peculiar abundance pattern that is indicative of it belonging to the class of metallic-line A stars (Am stars). The hallmark of such stars is that they have some stronger metallic lines (such as strontium) than are expected for stars of their effective temperatures (as measured by their, e.g., $\mathrm{H} \alpha$ line), but weaker lines in others, such as calcium, than expected for the same metallicity and effective temperature. In other words, the star does not appear to have a consistent metallicity given its effective temperature.

This leads to a classical definition of Am stars, which notes that the spectral type one deduces depends on the feature used for typing. Because A stars in general have metallic lines that increase in strength toward later type, a spectral type based on some metal lines that show enhancement will lead to a spectral type for an Am star that is "too late" compared to the Balmer line spectral type. Similarly, because A stars have Ca II K lines that increase in strength toward later type, the calcium deficiency for Am stars will lead to a calcium spectral type that is "too early." Thus a classical definition of Am stars is a range of spectral types from these methods of at least five 

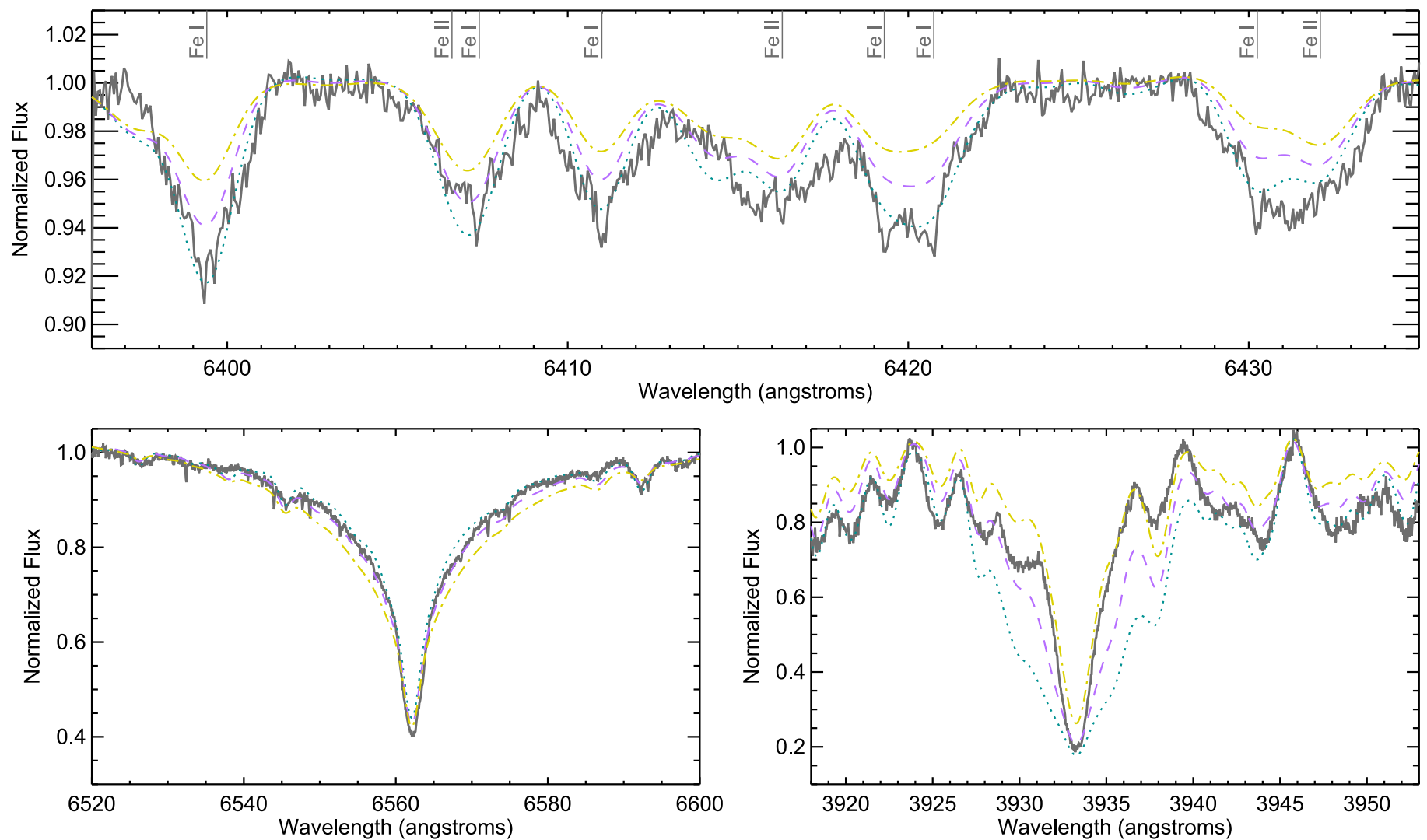

Figure 8. Spectrum of KELT-19A (gray solid line) and three PHOENIX model atmospheres (colored lines; Husser et al. 2013), overplotted for illustration (i.e., not fit to the data). All models were generated with $\log g_{\star}=4.124(\mathrm{cgs})$ and $[\mathrm{Fe} / \mathrm{H}]=+0.5$ and were broadened to $84.8 \mathrm{~km} \mathrm{~s}^{-1}$ rotation, but have temperatures of $7000 \mathrm{~K}$ (blue-green dotted), $7500 \mathrm{~K}$ (purple dashed), and $8000 \mathrm{~K}$ (yellow dash-dotted). Bottom left: the $\mathrm{H} \alpha$ profile is consistent with a $7500 \mathrm{~K}$ atmosphere, like we find in the spectroscopy and the global fit. Top: iron lines are enhanced, and therefore more consistent with a cooler $(7000 \mathrm{~K})$ atmosphere. Bottom right: the Ca II $\mathrm{K}$ line is weaker than expected, with a profile similar to that of the $8000 \mathrm{~K}$ atmosphere. A spectral type that is "too late" in metals and a Ca II K spectral type that is "too early" for the Balmer line spectral type is a hallmark of Am stars because of their photospheric metal enhancement and calcium deficiency.

subtypes. This is demonstrated in the spectrum of KELT-19A in Figures 8 and 9.

In Figure 8, the top panel shows iron lines, the bottom left panel shows $\mathrm{H} \alpha$, and the bottom right panel shows the Ca II $\mathrm{K}$ line. In each panel, the black line is the observed KELT-19A spectrum, and the thin colored lines are three PHOENIX model atmospheres. Each has the estimated $\log g_{\star}, v \sin I_{*}$, and $[\mathrm{Fe} / \mathrm{H}]=+0.5$ of KELT-19A. The blue-green dotted, purple dashed, and yellow dash-dotted lines correspond to 7000, 7500 , and $8000 \mathrm{~K}$, respectively. ${ }^{41}$ One can see that the $7000 \mathrm{~K}$ model (blue-green dotted line) is most appropriate for the metal lines, whereas the $\mathrm{H} \alpha$ is most consistent with our adopted temperature ( $\sim 7500 \mathrm{~K}$; purple dashed line), and the Ca II $\mathrm{K}$ line profile is most consistent with a hotter star $(8000 \mathrm{~K}$; yellow dash-dotted line). We also note that solar metallicity PHOENIX models all yield Fe II lines that are too weak at any temperature, which provides additional evidence that the photospheric metallicity is enhanced, as hinted at in the TODCOR analysis of Section 2.4.5.

To provide a more detailed spectral type for KELT-19, we compare its spectrum to a sequence of observed spectra ranging from A3V to F3V. All of these spectra were observed by TRES and reduced in the same way as KELT-19, which minimizes systematic bias, e.g., due to continuum normalization. Because each star has a different projected rotational line broadening, we measure it for each star and convolve the normalized

\footnotetext{
${ }^{41}$ None of these are fits; they are merely overplotted for illustration.
}

spectrum with a Gaussian with a width appropriate to produce a total broadening (rotational, instrumental, and artificial) of $100 \mathrm{~km} \mathrm{~s}^{-1}$. We compare KELT-19 to the spectral sequence and identify the spectral types that provide the best match to the Ca II K, H $\alpha$, and metal lines of KELT-19, and we illustrate this in Figure 9. While the Sr II line does not show any obvious enhancement, as might be expected for an Am star, this is not entirely surprising: the abundance anomalies in Am stars are negatively correlated with rotation so that those rotating as rapidly as KELT-19 are less anomalous; and the relatively rapid rotation of KELT-19 results in the Sr II line blending with at least three other lines of similar strength, so that the strength of any anomaly is diluted. Nevertheless, the other features in the spectrum are consistent with an Am star. An A5V star is an excellent match for the Ca II $\mathrm{K}$ line, an A7V star for the $\mathrm{H} \alpha$ profile, and an F2V star is the best match for the strength of the metal lines, resulting in a range of spectral types of $\sim \mathrm{A} 5$ to $\sim$ F2. We therefore conclude that KELT-19A meets the classical definition of an Am star, with a spectral type of "Am kA5 hA7 mF2 V."

\section{Host Star Properties}

Table 3 lists various properties and measurements of KELT19 collected from the literature and derived in this work. The data from the literature include $B V$ and gri photometry from Henden et al. (2015), optical fluxes in the $B_{\mathrm{T}}$ and $V_{\mathrm{T}}$ passbands from the Tycho-2 catalog (Høg et al. 2000), near-infrared (IR) 


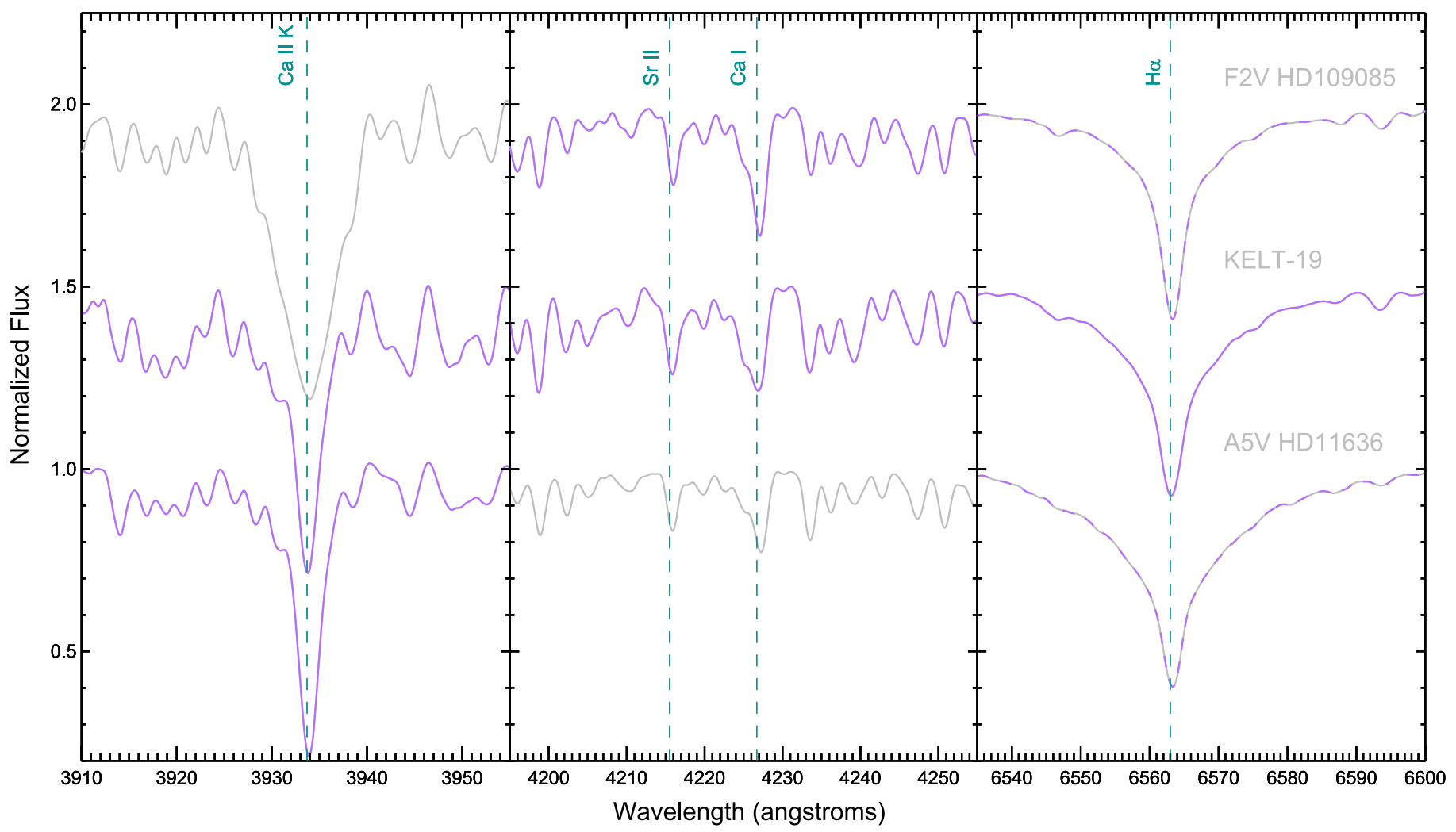

Figure 9. Spectrum of KELT-19 (middle row, purple) and two spectral standard stars-F2V HD109085 (top) and A5V HD11636 (bottom). All spectra were obtained by TRES and broadened so that the total broadening (combined rotational, instrumental, and artificial) is $100 \mathrm{~km} \mathrm{~s}^{-1}$. Each panel shows a feature or features important for determining spectral type, and we color the matching spectrum purple to indicate a match (or dashed purple to indicate a partial match). The Ca II $\mathrm{K}$ profile (left) of KELT-19 is an excellent match for that of the A5V star. The $\mathrm{H} \alpha$ profile (right) is broader than the F2V, narrower than the A5V, and matches an A7V spectrum well (not pictured). The strength of the metal lines (middle) match the F2V spectrum, with the exception of Ca I, which is much weaker in KELT-19. Sr II, which is expected to be enhanced in Am stars, does not appear significantly stronger in KELT-19, but it is blended with many other lines because of the star's rapid rotation.

fluxes in the $J, H$, and $K_{\mathrm{S}}$ passbands from the 2MASS Point Source Catalog (Cutri et al. 2003; Skrutskie et al. 2006), nearand mid-IR fluxes in four WISE passbands (Wright et al. 2010; Cutri et al. 2012), and distance and proper motions from Gaia (Gaia Collaboration et al. 2016).

\section{Analysis and Results}

\subsection{SED Analysis}

We performed a fit to the broadband SED of KELT-19 in order to obtain constraints on stellar parameters for use in the global system fit. We assembled the available broadband photometry from extant catalogs, with measurements spanning over the wavelength range $0.4-22 \mu \mathrm{m}$ (see Figure 10 and Table 3).

For the fitting, we used the stellar atmosphere models of Kurucz (1992), where the free parameters are the effective temperature $\left(T_{\text {eff }}\right)$, the extinction $\left(A_{V}\right)$, and the distance $\left(d_{\star}\right)$. In principle, the atmosphere models also depend on metallicity $([\mathrm{Fe} / \mathrm{H}])$ and surface gravity $\left(\log g_{\star}\right)$, but we do not have strong independent constraints on these, and in any event, they are of secondary importance to $T_{\text {eff }}$ and $A_{V}$. Thus we assumed a mainsequence $\log g_{\star} \approx 4.0$ and a solar $[\mathrm{Fe} / \mathrm{H}]$. For $A_{V}$, we restricted the maximum permitted value to be that of the full line-of-sight extinction from the dust maps of Schlegel et al. (1998). We also ran the fit with $[\mathrm{Fe} / \mathrm{H}]=+0.5$, and the result was not significantly different than the solar metallicity result.
Importantly, the high-resolution imaging (see Section 2.3) revealed another faint star, sufficiently close to KELT-19 that it can be assumed to contaminate the broadband photometry. Therefore, we performed the fit with two components, assuming (for the purposes of the fit) the same $A_{V}$ and $d_{\star}$ for both, and we adopted as additional constraints the flux ratios determined from the adaptive optics imaging and from the spectroscopic analysis: $F_{B} / F_{A}=0.0270 \pm 0.0034$ in the range $5200 \pm 150 \AA$, $\Delta J=2.50 \pm 0.06$, and $\Delta K_{S}=2.045 \pm 0.030$. This introduces one additional fit parameter, namely, the ratio of stellar radii $\left(R_{B} / R_{A}\right)$ that effectively sets the relative bolometric fluxes of the two stars.

The best-fit model shown in Figure 10 has a reduced $\chi^{2}$ of 0.66. We find $A_{\mathrm{V}}=0.03 \pm 0.03, T_{\text {eff }_{A}}=7500 \pm 200 \mathrm{~K}$, $T_{\text {eff } B}=5200 \pm 100 \mathrm{~K}, d_{\star}=255 \pm 15 \mathrm{pc}$, and $R_{2} / R_{1}=$ $0.46 \pm 0.03$. We note that the quoted statistical uncertainties on $A_{\mathrm{V}}$ and $T_{\text {eff }}$ are likely to be slightly underestimated because we have not accounted for the uncertainty in $\log g_{\star}$ or $[\mathrm{Fe} / \mathrm{H}]$. We also note, however, that the inferred $d_{\star}$ obtained here is fully consistent with that from the Gaia parallax (after correction for the systematic offset of -0.18 mas determined by Stassun \& Torres 2016), and moreover, the inferred properties of the secondary star are consistent with those of the observed secondary spectrum (see Section 2.4).

The two-component SED fit also permits determination of the amount of contaminating flux from the companion in the observed transit at each wavelength. This is accounted for in the global solution, as discussed in Section 4.4. 
Table 3

Literature Properties for KELT-19

\begin{tabular}{|c|c|c|c|}
\hline \multirow{4}{*}{ Other Names } & \multicolumn{3}{|l|}{$\mathrm{BD}+071721$} \\
\hline & \multicolumn{3}{|l|}{ TYC 764-1494-1 } \\
\hline & \multicolumn{3}{|c|}{ 2MASS J07260228 + 0736569} \\
\hline & \multicolumn{3}{|l|}{ TIC 425206121} \\
\hline Parameter & Description & Value & References \\
\hline$\alpha_{\mathrm{J} 2000}$ & Right Ascension (R.A.) & $07^{\mathrm{h}} 26^{\mathrm{m}} 02^{\mathrm{s}} .2895$ & 1 \\
\hline$\delta_{\mathrm{J} 2000}$ & Declination (Decl.) & $+07^{\circ} 36^{\prime} 56^{\prime \prime} 834$ & 1 \\
\hline$B_{\mathrm{T}}$ & Tycho $B_{\mathrm{T}}$ mag. & $10.273 \pm 0.036$ & 2 \\
\hline$V_{\mathrm{T}}$ & Tycho $V_{\mathrm{T}}$ mag. & $9.899 \pm 0.035$ & 2 \\
\hline$B$ & APASS Johnson $B$ mag. & $10.201 \pm 0.030$ & 3 \\
\hline$V$ & APASS Johnson $V$ mag. & $9.885 \pm 0.040$ & 3 \\
\hline$g^{\prime}$ & APASS Sloan $g^{\prime}$ mag. & $10.163 \pm 0.120$ & 3 \\
\hline$r^{\prime}$ & APASS Sloan $r^{\prime}$ mag. & $9.872 \pm 0.050$ & 3 \\
\hline$i^{\prime}$ & APASS Sloan $i^{\prime}$ mag. & $9.878 \pm 0.040$ & 3 \\
\hline$J$ & 2MASS $J$ mag. & $9.343 \pm 0.030$ & 4 \\
\hline$H$ & 2MASS $H$ mag. & $9.237 \pm 0.020$ & 4 \\
\hline$K$ & 2MASS $K$ mag. & $9.196 \pm 0.020$ & 4 \\
\hline WISE1 & WISE1 mag. & $9.138 \pm 0.022$ & 5 \\
\hline WISE2 & WISE2 mag. & $9.156 \pm 0.020$ & 5 \\
\hline WISE3 & WISE3 mag. & $9.132 \pm 0.035$ & 5 \\
\hline WISE4 & WISE4 mag. & $\geqslant 8.089$ & 5 \\
\hline$\mu_{\alpha}$ & 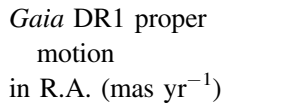 & $-3.706 \pm 1.126$ & 6 \\
\hline$\mu_{\delta}$ & 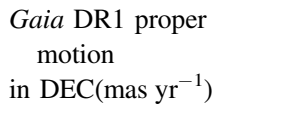 & $-1.303 \pm 1.226$ & 6 \\
\hline$R V$ & $\begin{array}{l}\text { Systemic radial } \\
\text { velocity }\left(\mathrm{km} \mathrm{s}^{-1}\right)\end{array}$ & $-8.5 \pm 0.5$ & Section 2.4 .3 \\
\hline$v \sin i_{\star}$ & $\begin{array}{l}\text { Stellar rotational } \\
\text { velocity }\left(\mathrm{km} \mathrm{s}^{-1}\right)\end{array}$ & $84.8 \pm 2.0$ & Section 4.4 .4 \\
\hline Sp. Type ${ }_{A}$ & Primary Star Sp. Type & $\mathrm{A} 8 \mathrm{~V}$ & Section 2.4 .5 \\
\hline Sp. Type ${ }_{B}$ & $\begin{array}{l}\text { Secondary Star } \\
\text { Sp. Type }\end{array}$ & G9V-K1V & Section 2.4 .5 \\
\hline Age & Age (Gyr) & $1.1 \pm 0.1$ & Section 4.2 \\
\hline$\Pi$ & Gaia Parallax (mas) & $3.60 \pm 0.72$ & $6^{\mathrm{a}}$ \\
\hline$d_{\star G a i a}$ & Gaia-inferred dist. (pc) & $278_{-47}^{+69}$ & $6^{\mathrm{a}}$ \\
\hline$d_{\star \mathrm{SED}}$ & SED-inferred dist. (pc) & $255 \pm 15$ & Section 4.1 \\
\hline$A_{V}$ & Visual extinction (mag) & $0.03 \pm 0.03$ & Section 4.1 \\
\hline$U^{*}$ & Space motion $\left(\mathrm{km} \mathrm{s}^{-1}\right)$ & $14.6 \pm 0.9$ & Section 4.3 \\
\hline$V$ & Space motion $\left(\mathrm{km} \mathrm{s}^{-1}\right)$ & $17.6 \pm 1.3$ & Section 4.3 \\
\hline$W$ & Space motion $\left(\mathrm{km} \mathrm{s}^{-1}\right)$ & $0.2 \pm 1.4$ & Section 4.3 \\
\hline
\end{tabular}

Note.

${ }^{\text {a }}$ Gaia parallax after correcting for the systematic offset of -0.18 mas for an ecliptic latitude of $-14^{\circ}$ as described in Stassun \& Torres (2016).

References are (1) van Leeuwen (2007), (2) Høg et al. (2000), (3) Henden et al. (2015), (4) Cutri et al. (2003), (5) Cutri et al. (2013), (6) Gaia Collaboration et al. (2016) Gaia DR1 http://gea.esac.esa.int/archive/.

\subsection{Stellar Models and Age}

With $T_{\text {eff }}$ from the SED analysis and with an estimated $\log g_{\star}$ and $M_{\star}$ from the global analysis (see below), we can place KELT-19A in the Kiel diagram for comparison with theoretical stellar evolutionary models (Figure 11). The estimated system age using the final global fit parameters is $\approx 1.1 \mathrm{Gyr}$, with an approximate uncertainty of order 0.1 Gyr. The KELT-19

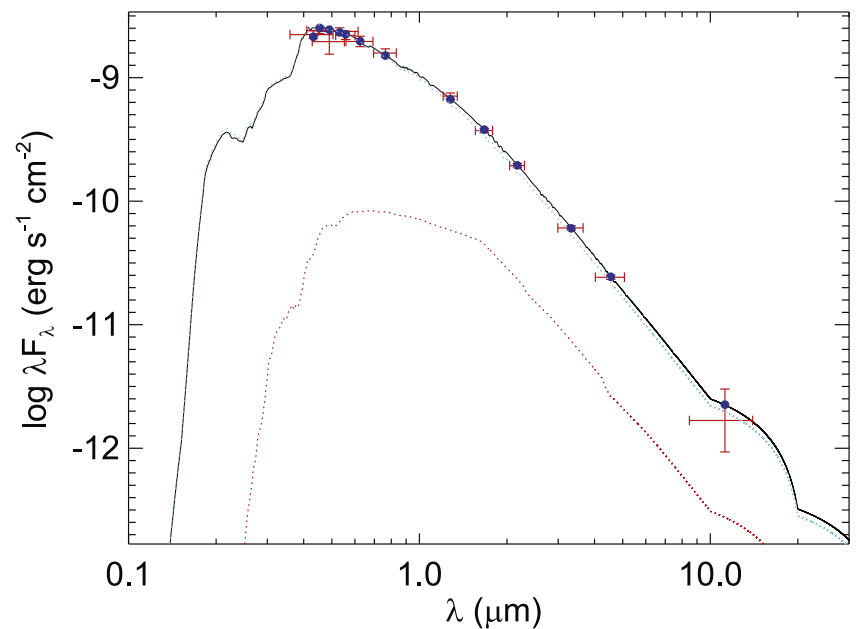

Figure 10. KELT-19 two-component SED. Crosses represent the measured fluxes of the two unresolved stars, with vertical bars representing the measurement uncertainties and the horizontal bars representing the width of the bandpass. The blue dots are the predicted passband-integrated fluxes of the best-fit theoretical SED corresponding to our observed photometric bands. The black solid, blue dotted, and red dotted curves represent the best-fit twocomponent KELT-19A and KELT-19B stellar atmospheres, respectively, from Kurucz (1992; see the text).

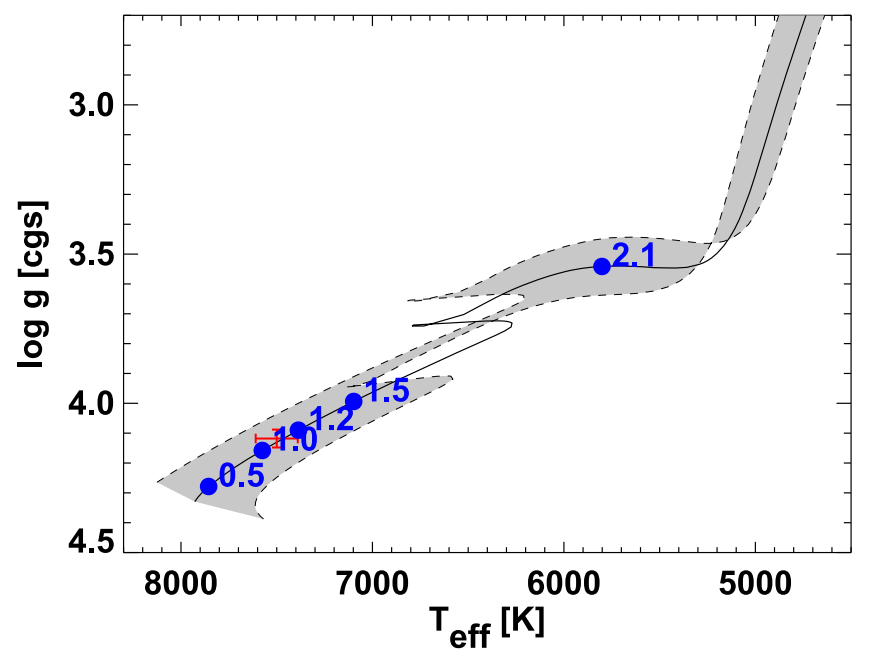

Figure 11. Evolution of the KELT-19A system in the Kiel diagram. The red cross represents the KELT-19A parameters from the final global fit. The black curve represents the theoretical evolutionary track for a star with the mass and metallicity of KELT-19A, and the gray swath represents the uncertainty on that track based on the uncertainties in mass and metallicity. Nominal ages in Gyr are shown as blue dots.

system is more than halfway through its main-sequence lifetime, but is at a stage of evolution well before the "blue hook" transition to the subgiant and eventual red giant evolutionary phases.

\subsection{UVW Space Motion}

We determine the 3D space velocity of KELT-19 in the usual $(U, V, W)$ coordinates in order to determine the Galactic population to which it belongs. We used a modification of the IDL routine GAL_UVW, which is itself based on the method of Johnson \& Soderblom (1987). We adopt the Gaia proper motions as listed in Table 3, the SED-inferred distance $255 \pm 15 \mathrm{pc}$, and the absolute radial velocity as 
determined from TRES spectroscopy of $-8.5 \pm 0.5 \mathrm{~km} \mathrm{~s}^{-1}$. We find that KELT-19 has $U, V, W$ space motion of $(U, V, W)=(14.6 \pm 0.9,17.6 \pm 1.3,0.2 \pm 1.4) \mathrm{km} \mathrm{s}^{-1}$, in a coordinate system where positive $U$ is in the direction of the Galactic center, and using the Coşkunoğlu et al. (2011) determination of the solar motion with respect to the local standard of rest. These values yield a $99.2 \%$ probability that the KELT-19 binary system is in the thin disk, according to the classification scheme of Bensby et al. (2003), as expected for its age and spectral type.

\subsection{Global System Fit}

We determined the physical and orbital parameters of the KELT-19A system by jointly fitting eight transit light curves, 7 TRES and 14 HJST out-of-transit RVs, and a TRES Doppler tomographic data set (see Section 2.4). To perform the global fit, we used MULTI-EXOFAST (MULTIFAST hereafter), which is a custom version of the public software package EXOFAST (Eastman et al. 2013). MULTIFAST first performs an AMOEBA (Nelder \& Mead 1965) best fit to each of the RV and light curve data sets individually to determine uncertainty scaling factors. The uncertainties are scaled such that the probability that the $\chi^{2}$ for a data set is higher than the value we achieved, $P\left(>\chi^{2}\right)$, is 0.5 , to ensure the resulting parameter uncertainties are roughly accurate. The resulting RV uncertainty scaling factors are 1.22 and 1.13 for the TRES and HJST velocities, respectively. The uncertainties of the DT observations were scaled by 1.0. Finally, MULTIFAST performs a joint AMOEBA model fit to all of the data sets and executes a Markov chain Monte Carlo (MCMC), starting at the global best-fit values, to determine the median and $68 \%$ confidence intervals for each of the physical and orbital parameters. MULTIFAST provides the option to include the Yonsei-Yale (YY) stellar model constraints (Demarque et al. 2004) or the Torres empirical constraints (Torres et al. 2010) to break the wellknown degeneracy between $M_{\star}$ and $R_{\star}$ for single-lined spectroscopic eclipsing systems. Siverd et al. (2012) provides a more detailed description of MULTIFAST, except for the Doppler tomographic model, which is described in Gaudi et al. (2017).

\subsubsection{Light-curve Detrending and Deblending}

We use AIJ to determine the best detrending parameter data sets to include in the MULTIFAST global model by finding the AMOEBA best fit of a Mandel \& Agol (2002) exoplanet transit model to the transit photometry plus linear combination(s) of detrending data $\operatorname{set}(\mathrm{s})$. Up to two detrending data sets were selected per light curve based on the largest reductions in the Bayesian information criterion (BIC) calculated by AIJ from the model fits with and without the detrending data set included. A detrending data set was not included unless it reduced the $\mathrm{BIC}$ by $>2.0$, resulting in some light curves with only one detrending data set. The final detrending data sets we chose for each light curve are listed in Table 1. It is important to emphasize that the AIJ-extracted raw differential light curves (i.e., not detrended) and the detrending data sets were inputs to MULTIFAST and were simultaneously fitted as a part of the global models.

As discussed in Sections 2.3 and 2.4.3, KELT-19A has a bound stellar secondary companion at a projected separation of 0 .'64. Because the secondary is blended in all follow-up
Table 4

Flux Contamination from SED Fit

\begin{tabular}{lc}
\hline \hline Band & $F_{\mathrm{B}} / F_{\mathrm{A}}$ \\
\hline $5200 \pm 150 \AA$ & 0.02782 \\
$\mathrm{U}$ & 0.02412 \\
$\mathrm{~B}$ & 0.03389 \\
$\mathrm{~V}$ & 0.03710 \\
$\mathrm{R}$ & 0.04619 \\
$\mathrm{I}$ & 0.04693 \\
Sloan $g^{\prime}$ & 0.03961 \\
Sloan $r^{\prime}$ & 0.04469 \\
Sloan $i^{\prime}$ & 0.04867 \\
Sloan $z^{\prime}$ & 0.05261 \\
\hline
\end{tabular}

photometry apertures, the flux from the secondary must be taken into account to obtain the correct transit depth and planetary radius (e.g., Ciardi et al. 2015). As discussed in Section 4.1, the two-component SED fit permits determination of the amount of contaminating flux from the companion in the observed transit at each wavelength. The determined blend factors, $F 2 / F 1$, for all of the follow-up photometry filter bands are shown in Table 4 . The blend factors for each filter band were included in MULTIFAST as fixed values to adjust the transit depth in each filter to account for the blend.

\subsubsection{Gaussian and Uniform Priors}

We included Gaussian priors on the reference transit center time, $T_{0}$, and orbital period, $P$. To determine the prior values for the final global fits, we executed preliminary MULTIFAST global fits, including a transit-timing variation (TTV) parameter in the model for each light curve to allow the transit center time to vary from a linear ephemeris, and used priors $T_{0}=2457055.276 \pm 0.013$ $\mathrm{BJD}_{\mathrm{TDB}}$ and $P=4.611758 \pm 0.000053$ days derived from the KELT data. For these preliminary fits, we included the eight primary transit light curves and the DT data. We chose to include a circular orbit constraint and fixed the RV slope to zero for the model fits. The preliminary YY-constrained model fit resulted in a TTV-based linear ephemeris $T_{0}=2457281.249522 \pm 0.000359$ $\mathrm{BJD}_{\mathrm{TDB}}$ and $P=4.6117091 \pm 0.0000089$ days. These values were used as Gaussian priors in the final YY-constrained global model fit. The preliminary Torres-constrained model fit resulted in a TTV-based linear ephemeris $T_{0}=2457285.861243 \pm$ $0.000355 \mathrm{BJD}_{\mathrm{TDB}}$ and $P=4.6117094 \pm 0.0000090$ days. These values were used as Gaussian priors in the final Torresbased global model fit. Since the KELT- and TTV-based ephemerides are generally derived from independent data, we propagate forward the precise TTV-based ephemerides without concern for double-counting data.

We also included Gaussian priors on the stellar parameters $T_{\text {eff }}=7505 \pm 104 \mathrm{~K}$ and $[\mathrm{Fe} / \mathrm{H}]=0.0 \pm 0.5$ from the SED analysis in Section 4.1 and the stellar parameter analysis in Section 2.4 .5 and $v \sin I_{\star}=84.1 \pm 2.1 \mathrm{~km} \mathrm{~s}^{-1}$ and macroturbulent broadening of $3.4 \pm 2.0 \mathrm{~km} \mathrm{~s}^{-1}$ from the out-of-transit broadening profile. A prior was not imposed on $\log g_{\star}$, since the value derived from the light curve-based stellar density and our stellar radius constraints is expected to be more accurate than the spectroscopic (e.g., Mortier et al. 2013, 2014) or SEDbased $\log g_{\star}$.

We limited the range of certain parameters by including bounded uniform priors. We restricted the RV semi-amplitude to $K>1.0 \mathrm{~m} \mathrm{~s}^{-1}$. To prevent problems when interpolating 
values from the limb-darkening tables, we restricted the stellar parameters to $3500 \leqslant T_{\text {eff }}<20,000 \mathrm{~K},-2.0 \leqslant[\mathrm{Fe} / \mathrm{H}]<1.0$, and $2.0 \leqslant \log g_{\star}<5.0$. We inspected the corresponding posterior parameter distributions to ensure that there was no significant likelihood near the uniform prior boundaries.

\subsubsection{Global Model Configurations}

We examine the results of two global model configurations to explore the effects of YY-constrained and Torres-constrained global model fits on parameter posterior distributions. Since no RV orbit is detected, we force both models to have a circular orbit and an RV slope of zero. Since the Gaia distance error is greater than $10 \%$, we do not impose an empirical stellar radius constraint.

\subsubsection{Global Model Results}

We adopt the posterior median parameter values and uncertainties of the YY-constrained fit as the fiducial global model and compare to the results from the Torres-constrained global model. The posterior median parameter values and $68 \%$ confidence intervals for both final global models are shown in Table 5. The KELT-19Ab fiducial model indicates that the system has a host star with mass $M_{\star}=1.62 M_{\odot}$, radius $R_{\star}=1.830 R_{\odot}$, and effective temperature $T_{\text {eff }}=7,500 \mathrm{~K}$, and a planet with $T_{\mathrm{eq}}=1935 \mathrm{~K}$, and radius $R_{\mathrm{P}}=1.891 R_{\mathrm{J}}$. Because an $\mathrm{RV}$ orbit is not detected, we state $3 \sigma$ upper limits on all of the planet mass-related posterior parameter values. The planet mass of KELT-19Ab is constrained to be $<4.07 \mathrm{M}_{\mathrm{J}}$ with $3 \sigma$ significance.

In summary, we find that the YY and Torres stellar constraints result in system parameters that are well within $1 \sigma$.

\subsubsection{Transit-timing Variation Results}

We derive a precise linear ephemeris from the transit-timing data by fitting a straight line to all inferred transit center times. These times are listed in Table 6 and plotted in Figure 12. We find a best-fit linear ephemeris of $T_{0}=2457281.249537 \pm$ $0.000362 \mathrm{BJD}_{\mathrm{TDB}}, P_{\text {Transits }}=4.6117091 \pm 9.0 \times 10^{-6}$ days, with a $\chi^{2}$ of 20.9 and 6 degrees of freedom, resulting in $\chi_{r}^{2}=3.5$. While the $\chi_{r}^{2}$ is larger than one might expect, this is often the case in ground-based TTV studies, likely due to systematics in the transit data. Even so, all of the timing deviations are less than $3 \sigma$ from the linear ephemeris. Furthermore, note that the TTVs of the four simultaneous transit observations on epoch 27 range from $\sim-2 \sigma$ to $+3 \sigma$, indicating that the TTVs are likely due to light curve systematics. We therefore conclude that there is no convincing evidence for TTVs in the KELT-19Ab system. However, due to the limited number of full light curves included in this study, we suggest further transit observations of KELT-19Ab before ruling out TTVs.

\section{False-positive Analysis}

Despite the lack of a definitive measurement of the companion mass, we are confident that this system is truly a hot Jupiter transiting a late-A star. The evidence for this comes from several sources that we review briefly. However, we invite the reader to review papers by Bieryla et al. (2015), Zhou et al. (2016a, 2016b), and Hartman et al. (2015) for a more detailed explanation. The basic point is that the RV measurements, while not sufficiently precise to measure the mass of the transiting companion, do indicate that it is not a brown dwarf or a low-mass star, if it is indeed transiting the primary A star. On the other hand, we are confident that the companion is transiting the primary A star (rather than, say, the later spectral-type bound companion), because we see a Doppler tomographic signal with the expected amplitude, duration, and impact parameter inferred from the follow-up light curves. Of course, the first system to have been validated in this way was WASP-33b (Collier Cameron et al. 2010).

The Doppler tomographic observation eliminates the possibility of a blended eclipsing binary causing the transit signal. Even though the line profile derived from the least-squares deconvolution shows a spectroscopic companion blended with KELT-19A, the spectroscopic transit is seen crossing nearly the entirety of the rapidly rotating primary star's line profile (the TRES DT observations did not cover ingress), confirming that the planet is indeed orbiting KELT-19A. The summed flux underneath the Doppler tomographic shadow and the distance of closest approach of the shadow from the zero velocity at the center of the predicted transit time is consistent with both the photometric transit depth and impact parameter, suggesting that the photometric transit is not diluted by background stars, and is fully consistent with the spectroscopic transit.

Adaptive optics observations (Section 2.3) also show a nearby companion consistent in relative brightness with the TRES companion's relative brightness, but no other stars brighter than $\Delta \mathrm{Br}-\gamma<6$ with separation $>0$ ". 6 from KELT$19 \mathrm{~A}$ at $5 \sigma$ significance. Furthermore, the deblended follow-up observation transit depths are consistent across the optical and infrared bands, as indicated in Figure 2.

Finally, the planetary nature of KELT-19Ab is confirmed by the TRES and HJST RV measurements, which constrain the mass of the companion to be $\lesssim 4.1 \mathrm{M}_{\mathrm{J}}$ at $3 \sigma$ significance. This eliminates the possibility that the transiting companion is a stellar or brown-dwarf-mass object.

Thus we conclude that all the available evidence suggests that the most plausible interpretation is that KELT-19Ab is a Jupiter-sized planet transiting the late-A star TYC 764-1494-1 with a retrograde projected spin-orbit alignment (see Sections 2.4 .4 and 6.1), and with a late-G or early-K bound companion with a projected separation of $\approx 160 \mathrm{au}$.

We do note, however, that this was a particularly complicated case, one that may have easily been rejected as a false positive based simply on the double-lined nature of the line profiles (see Figure 5). KELT-19Ab therefore provides an important object lesson: transiting planets can indeed be found and definitively confirmed in initially unresolved binary systems. Indeed, such systems may provide important constraints on the emplacement of hot Jupiters, as the outer bound stellar companion can easily be responsible for Kozai-Lidov oscillations and so emplacement of hot Jupiters (Kozai 1962; Lidov 1962).

\section{Discussion}

Figure 13 shows host star effective temperature versus $V$ band magnitude for known transiting planets. Within $T_{\text {eff }}$ uncertainties, KELT-19A joins KELT-17, WASP-33, HAT-P57, and MASCARA-1 as having the third highest $T_{\text {eff }}$ of all known transiting hot-Jupiter host stars. With a host star luminosity of $\sim 9.5 L_{\odot}$ and an orbital period of $\sim 4.6$ days, the planet has a high equilibrium temperature of $T_{\mathrm{eq}} \sim 2000 \mathrm{~K}$, assuming zero albedo and perfect heat redistribution. With a $V$-band magnitude of 9.9, a high equilibrium temperature, and a 
Table 5

Global Fit Posterior Parameter Values for the KELT-19Ab System

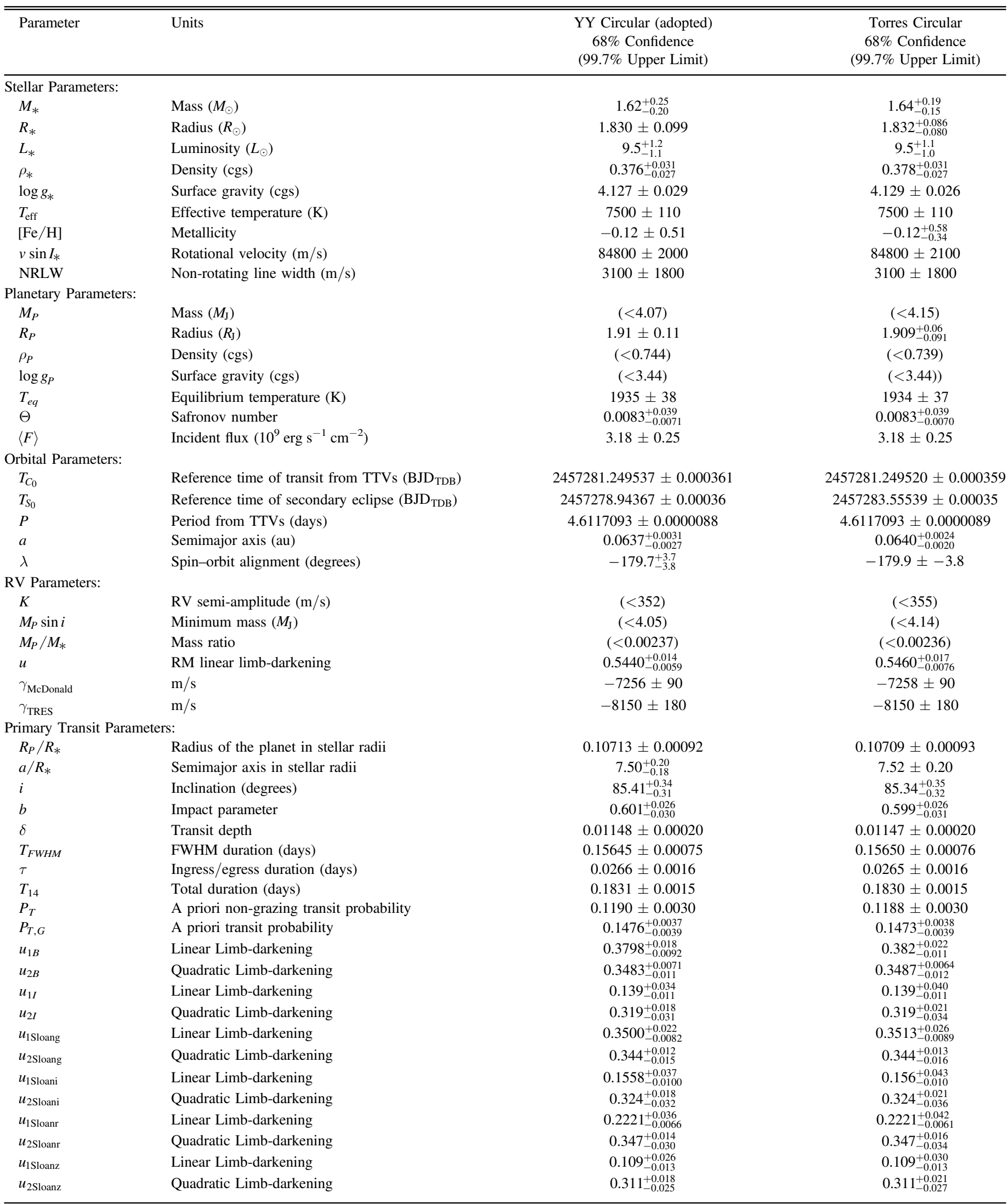




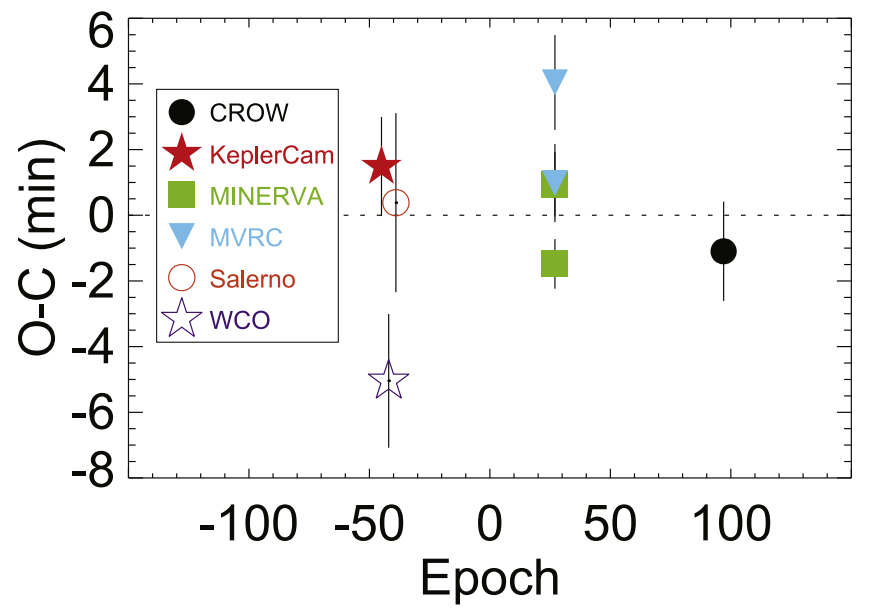

Figure 12. KELT-19Ab TTVs. All of the timing deviations are less than $3 \sigma$ from the linear ephemeris. The transit center times of the four transits on epoch 27 range from $\sim-2 \sigma$ to $+3 \sigma$, indicating that the TTVs are likely due to lightcurve systematics rather than astrophysical influences.

Table 6

Transit Times for KELT-19Ab

\begin{tabular}{lcrrrl}
\hline \hline Epoch & $T_{C}$ & $\begin{array}{r}\sigma_{T_{C}} \\
\left(\mathrm{BJD}_{\mathrm{TDB}}\right)\end{array}$ & \multicolumn{1}{c}{$\begin{array}{c}\text { O-C } \\
(\mathrm{s})\end{array}$} & \multicolumn{1}{c}{$(\mathrm{s})$} & \multicolumn{1}{c}{ O-C } \\
$\left(\sigma_{T_{C}}\right)$ & Telescope \\
\hline-45 & 2457073.723660 & 90 & 89.10 & 0.98 & KeplerCam \\
-42 & 2457087.554255 & 122 & -302.48 & -2.48 & WCO \\
-39 & 2457101.393149 & 163 & 22.97 & 0.14 & Salerno \\
27 & 2457405.764653 & 45 & -88.84 & -1.97 & MINERVA \\
27 & 2457405.766335 & 59 & 56.49 & 0.96 & MINERVA \\
27 & 2457405.768490 & 86 & 242.68 & 2.80 & MVRC \\
27 & 2457405.766362 & 71 & 58.82 & 0.82 & MVRC \\
97 & 2457728.584553 & 90 & -65.88 & -0.73 & CROW \\
\hline
\end{tabular}

likely large scale height, it is an excellent target for detailed follow-up and characterization. Because KELT-19A is an A star, the planet receives a higher amount of high-energy radiation than the majority of known transiting planet systems, which may lead to significant atmospheric ablation (MurrayClay et al. 2009).

\subsection{Spin-Orbit Misalignment}

Although we have measured the sky-projected spin-orbit misalignment $\lambda$, we cannot measure the full 3D spin-orbit misalignment $\psi$ because we do not know the inclination angle of the stellar rotation axis with respect to the line of sight, $I_{*}$. We can, however, set limits upon $\psi$. First, we follow Iorio (2011) and limit $I_{*}$, and therefore $\psi$, by requiring that KELT19A must rotate at less than break-up velocity. Doing so, we find that at $1 \sigma$ confidence, $19.7<I_{*}<160^{\circ} .0$ and $105^{\circ}<\psi<180^{\circ}$. We can, however, use the possible Am star nature of KELT-19A to set somewhat stricter limits upon $I_{*}$ and $\psi$. Although physically KELT-19A must have an equatorial rotation velocity of $v_{\mathrm{eq}}<250 \mathrm{~km} \mathrm{~s}^{-1}$ to avoid break-up, empirically, Am stars are not observed to have rotation velocities greater than $\sim 150 \mathrm{~km} \mathrm{~s}^{-1}$. If we instead require that KELT-19A have $v_{\mathrm{eq}}<150 \mathrm{~km} \mathrm{~s}^{-1}$, we obtain limits of $33^{\circ} .5<I_{*}<146^{\circ} .5$ and $119^{\circ}<\psi<180^{\circ}$.

KELT-19Ab continues the trend of hot Jupiters around A stars to have a wide range of sky-projected spin-orbit misalignments. Of the eight A-star-hosted hot Jupiters with measured spin-orbit

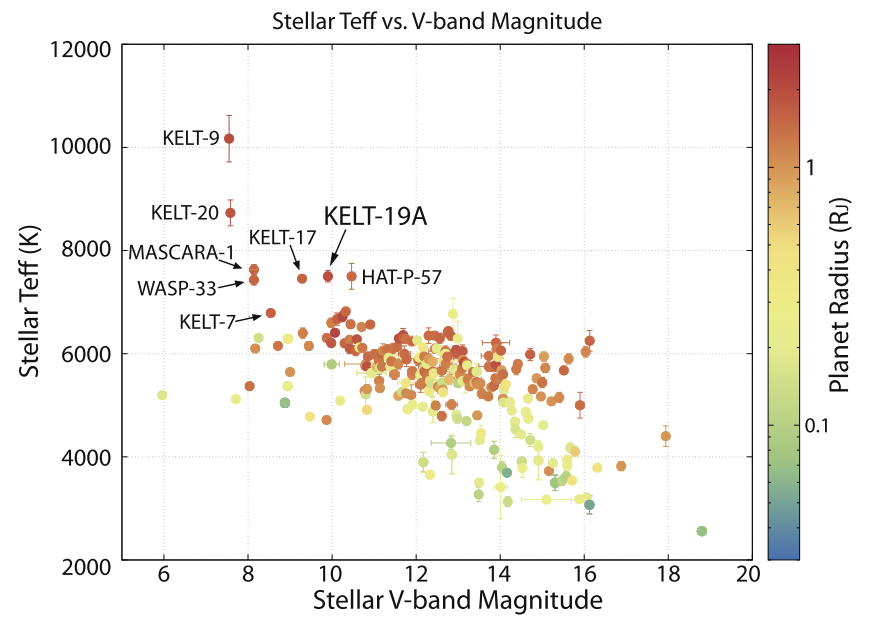

Figure 13. Population of transiting exoplanets based on the host star's $V$-band magnitude and effective temperature $\left(T_{\text {eff }}\right)$, with colors indicating the radius of the planet in $R_{\mathrm{J}}$. Within $T_{\text {eff }}$ uncertainties, KELT-19A joins KELT-17, HAT-P57 , and WASP-33 as having the third highest $T_{\text {eff }}$ of all known transiting hotJupiter host stars. These data, except for KELT-19A and KELT-20, were extracted from the NASA Exoplanet Database (https://exoplanetarchive. ipac.caltech.edu).

misalignments to date, one is on a prograde, well-aligned orbit (KELT-20b/MASCARA-2b: Lund et al. 2017; Talens et al. 2017b); two have misaligned prograde orbits (Kepler$13 \mathrm{Ab}$ and MASCARA-1b: Johnson et al. 2014; Talens et al. 2017a); one is on a prograde orbit with an unclear degree of misalignment (HAT-P-57b: Hartman et al. 2015); one is on a near-polar orbit (KELT-9b: Gaudi et al. 2017); two are on misaligned retrograde orbits (WASP-33b and KELT-17b: Collier Cameron et al. 2010; Zhou et al. 2016a); and, now, KELT-19Ab is on a near-antialigned retrograde orbit. Qualitatively, the distribution of A-star hot-Jupiter spin-orbit misalignments appears consistent with isotropic, but detailed investigation of this distribution will require a larger sample of planets.

\subsection{Tidal Evolution and Irradiation History}

Following Penev et al. (2014), we model the orbital evolution of KELT-19Ab due to the dissipation of the tides raised by the planet on the the host star under the assumption of a constant phase lag. The starting configuration of the system was tuned to reproduce the currently observed system parameters (Table 5) at the assumed system age of $1.1 \mathrm{Gyr}$ (see Section 4.2). The evolution model includes the effects of the changing stellar radius and luminosity following the YY circular stellar model with mass and metallicity as given in Table 5. No effects of the stellar rotation have been included in the calculation, since the star is observed to counter-rotate with respect to the orbit. In a retrograde configuration, tidal coupling always acts to remove energy and angular momentum from the planet, and as a result, under the assumption of a constant phase lag, the evolution is indistinguishable from that of a nonrotating host star.

Orbital and stellar irradiation evolutions are shown in Figure 14 for a range of stellar tidal quality factors $\left(Q_{*}^{\prime}=10^{5}, 10^{6}\right.$, and $\left.10^{7}\right)$, where $Q_{*}^{\prime-1}$ is the product of the tidal phase lag and the Love number. We find that the insolation received by the planet is well above the empirical inflation irradiation threshold of $\sim 2 \times 10^{8} \mathrm{erg} \mathrm{s}^{-1} \mathrm{~cm}^{-2}$ (Demory \& Seager 2011) for the entire main-sequence existence of the star (bottom panel of Figure 14). 

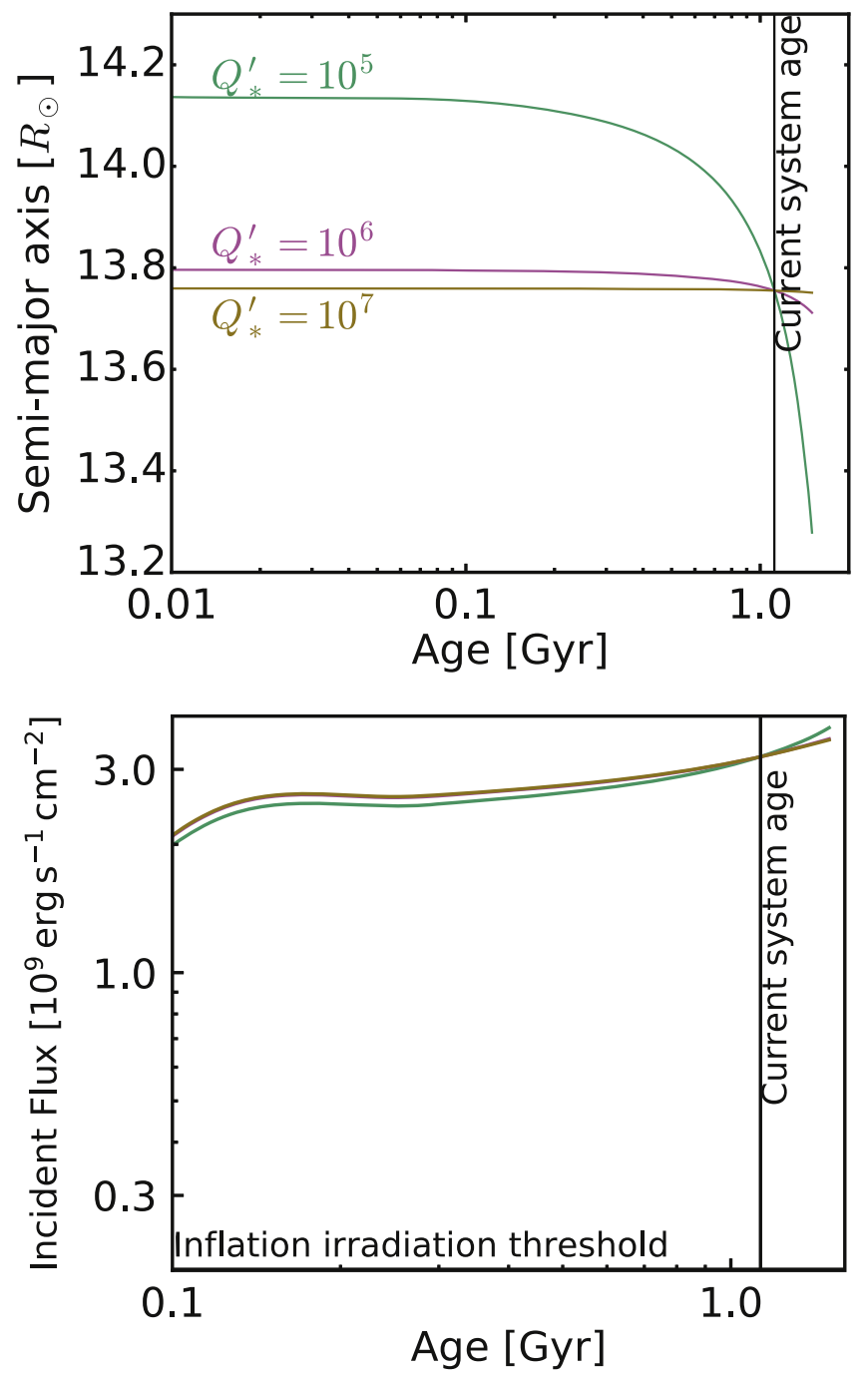

Figure 14. (Top) Orbital semimajor axis history of KELT-19Ab modeled for a range of stellar tidal quality factors, $Q_{*}^{\prime}$, where $Q_{*}^{\prime-1}$ is the product of the tidal phase lag and the Love number. The black vertical line marks the current system age of 1.1 Gyr. (Bottom) Irradiation history of KELT-19Ab modeled for a range of stellar tidal quality factors. The black horizontal line nearly coincident with the $x$-axis marks the inflation irradiation threshold of $\approx 2 \times 10^{8} \mathrm{erg} \mathrm{s}^{-1} \mathrm{~cm}^{-2}$ (Demory \& Seager 2011).

We consider a wide range of $Q_{*}^{\prime}$ because of the wide range of proposed mechanisms for tidal dissipation in current theoretical models and the conflicting observational constraints backing those models, especially for stars that may have surface convective zones (see the review by Ogilvie 2014 and references therein). Furthermore, because the dependence on stellar mass and tidal frequency is different for the different proposed mechanisms, we make the simplifying assumption that $Q_{*}^{\prime}$ remains constant over the life of the star. However, with multi-year baselines, it may be possible in the future to empirically constrain the lower limit on $Q_{*}^{\prime}$ for KELT-19Ab via precise measurements of the orbital period time decay (cf. Hoyer et al. 2016).

Finally, note that this model does not account in any way for the larger-distance Type II or scattering-induced migration that KELT-19Ab and other hot Jupiters likely undergo. It considers only the close-in migration due to tidal friction.

\section{Conclusion}

KELT-19 consists of a hierarchical triple system of an Am star that is being transited by a $P \sim 4.6$ day hot Jupiter with a mass of $\lesssim 4 M_{\mathrm{J}}$. The planet is highly inflated and highly irradiated, with a radius of $\simeq 2 R_{\mathrm{J}}$, and an equilibrium temperature of $T_{\text {eq }} \sim 2000 \mathrm{~K}$. It is also on a retrograde orbit with projected spin-orbit alignment of $\lambda \sim-180$ degrees. Finally, the primary A star (KELT-19A) and hot Jupiter (KELT-19Ab) are orbited by an outer bound stellar G9V/K1V companion (KELT-19B) with a projected separation of $\sim 160$ au.

In many ways, KELT-19 is one of the most unusual transiting hot-Jupiter systems yet discovered. First, the primary star (KELT19A) and planet host is an Am (metallic line-enhanced) star. To the best of our knowledge, this is the only such star known to host a transiting hot Jupiter. ${ }^{42}$ As is the case for other Am stars, KELT-19A rotates slowly compared to stars of similar effective temperature. Although the presence of a nearby stellar companion is usually invoked to explain both the slower rotation and peculiar abundance patterns of Am stars, the stellar companion KELT-19B seems too distant to cause significant tidal braking. Furthermore, the planetary companion (KELT-19Ab) is likely too low in mass to sufficiently slow the rotation of its host star, KELT-19A (Matsumura et al. 2010). Thus, the slow rotation of KELT-19A is probably either primordial or was induced by a more efficient tidal braking mechanism than expected.

Finally, we note that the confirmation of KELT-19Ab provides an important object lesson for future transit surveys. The initial line-spread function exhibited two peaks: a broad peak due to the rapidly rotating A star, and a narrower peak due to the more slowly rotating (but bound) blended late-G/early-K companion. Without careful analysis, such multiple-star systems may be spuriously rejected as false positives. Generally, we suggest that multi-lined systems not be immediately discarded unless the line of the blended secondary shows relative motion that is consistent with the photometric ephemeris of the transit event, in which case the secondary is likely one component of a eclipsing binary whose eclipses are being diluted by the primary. In this case, our analysis revealed the presence of a genuine transiting hot Jupiter orbiting an A-type star in a hierarchical triple system.

This project makes use of data from the KELT survey, including support from The Ohio State University, Vanderbilt University, and Lehigh University, along with the KELT followup collaboration. Work performed by J.E.R. was supported by the Harvard Future Faculty Leaders Postdoctoral fellowship. D.J. S. and B.S.G. were partially supported by NSF CAREER Grant AST-1056524. Work by S.V.Jr. is supported by the National Science Foundation Graduate Research Fellowship under Grant No. DGE-1343012. Work by G.Z. is provided by NASA through Hubble Fellowship grant HST-HF2-51402.001-A awarded by the Space Telescope Science Institute, which is operated by the Association of Universities for Research in Astronomy, Inc., for NASA, under contract NAS 5-26555. This paper includes data taken at The McDonald Observatory of The University of Texas at Austin. This work has made use of NASA's Astrophysics Data System, the Extrasolar Planet Encyclopedia, the NASA Exoplanet Archive, the SIMBAD database operated at CDS, Strasbourg,

\footnotetext{
${ }^{42}$ However, see Grenier et al. (1999), who suggest that WASP-33 may be an Am star, although Collier Cameron et al. (2010) note that "No obvious Am characteristics are visible in this spectrum other than slightly weak Ca II H\&K lines."
} 
France, and the VizieR catalog access tool, CDS, Strasbourg, France. We make use of Filtergraph, an online data visualization tool developed at Vanderbilt University through the Vanderbilt Initiative in Data-intensive Astrophysics (VIDA). We also used data products from the Widefield Infrared Survey Explorer, which is a joint project of the University of California, Los Angeles; the Jet Propulsion Laboratory/California Institute of Technology, which is funded by the National Aeronautics and Space Administration; the Two Micron All Sky Survey, which is a joint project of the University of Massachusetts and the Infrared Processing and Analysis Center/California Institute of Technology, funded by the National Aeronautics and Space Administration and the National Science Foundation; and the European Space Agency (ESA) mission Gaia (http://www. cosmos.esa.int/gaia), processed by the Gaia Data Processing and Analysis Consortium (DPAC, http://www.cosmos.esa.int/ web/gaia/dpac/consortium). Funding for the DPAC has been provided by national institutions, in particular the institutions participating in the Gaia Multilateral Agreement. MINERVA is a collaboration among the Harvard-Smithsonian Center for Astrophysics, The Pennsylvania State University, the University of Montana, and the University of New south Wales. MINERVA is made possible by generous contributions from its collaborating institutions and Mt. Cuba Astronomical Foundation, The David \& Lucile Packard Foundation, National Aeronautics and Space Administration (EPSCOR grant NNX13AM97A), The Australian Research Council (LIEF grant LE140100050), and the National Science Foundation (grants 1516242 and 1608203). Any opinions, findings, and conclusions or recommendations expressed are those of the author and do not necessarily reflect the views of the National Science Foundation. This work was partially supported by funding from the Center for Exoplanets and Habitable Worlds. The Center for Exoplanets and Habitable Worlds is supported by the Pennsylvania State University, the Eberly College of Science, and the Pennsylvania Space Grant Consortium.

\section{ORCID iDs}

Robert J. Siverd (1) https://orcid.org/0000-0001-5016-3359 Karen A. Collins 누 https://orcid.org/0000-0001-6588-9574 Samuel N. Quinn (1) https://orcid.org/0000-0002-8964-8377 B. Scott Gaudi 1 i https://orcid.org/0000-0003-0395-9869 Keivan G. Stassun (1) https://orcid.org/0000-0002-3481-9052 Marshall C. Johnson (1) https://orcid.org/0000-00025099-8185

Allyson Bieryla iㅏ https://orcid.org/0000-0001-6637-5401

David W. Latham (1) https://orcid.org/0000-0001-9911-7388

David R. Ciardi (1) https://orcid.org/0000-0002-5741-3047

Joseph E. Rodriguez (i) https://orcid.org/0000-0001-

8812-0565

Kaloyan Penev (1) https://orcid.org/0000-0003-4464-1371

Marc Pinsonneault (1) https://orcid.org/0000-0002-7549-7766

Joshua Pepper (1) https://orcid.org/0000-0002-3827-8417

Jason D. Eastman (1) https://orcid.org/0000-0003-3773-5142

John F. Kielkopf (1) https://orcid.org/0000-0003-0497-2651

Michael L. Calkins (1) https://orcid.org/0000-0002-2830-5661 Courtney D. Dressing (i) https://orcid.org/0000-00018189-0233

Rahul Patel (1) https://orcid.org/0000-0002-5025-6827

Thomas G. Beatty (1) https://orcid.org/0000-0002-9539-4203

Michael B. Lund (ㄱ) https://orcid.org/0000-0003-2527-1598
Jonathan Labadie-Bartz (1) https://orcid.org/0000-00022919-6786

Knicole D. Colón (i) https://orcid.org/0000-0001-8020-7121

David James (1) https://orcid.org/0000-0001-5160-4486

Jason T. Wright (10 https://orcid.org/0000-0001-6160-5888

Robert A. Wittenmyer (1) https://orcid.org/0000-00019957-9304

Samson A. Johnson 주 https://orcid.org/0000-0001-9397-4768

Eric L. N. Jensen (1) https://orcid.org/0000-0002-4625-7333

David H. Cohen (1ㄴ) https://orcid.org/0000-0003-2995-4767

Kim K. McLeod (i) https://orcid.org/0000-0001-9504-1486

Matthew T. Penny (1) https://orcid.org/0000-0001-7506-5640

Steven Villanueva, Jr. (1) https://orcid.org/0000-0001-

6213-8804

Christopher Stockdale (i) https://orcid.org/0000-0003-

2163-1437

Thiam-Guan Tan (1) https://orcid.org/0000-0001-5603-6895

\section{References}

Abt, H. A., \& Levy, S. G. 1985, ApJS, 59, 229

Abt, H. A., \& Morrell, N. I. 1995, ApJS, 99, 135

Alonso, R., Brown, T. M., Torres, G., et al. 2004, ApJL, 613, L153

Bakos, G. Á, Noyes, R. W., Kovács, G., et al. 2007, ApJ, 656, 552

Bensby, T., Feltzing, S., \& Lundström, I. 2003, A\&A, 410, 527

Bieryla, A., Collins, K., Beatty, T. G., et al. 2015, AJ, 150, 12

Casagrande, L., Ramírez, I., Meléndez, J., Bessell, M., \& Asplund, M. 2010, A\&A, 512, A54

Ciardi, D. R., Beichman, C. A., Horch, E. P., \& Howell, S. B. 2015, ApJ, 805,16

Claret, A. 2004, A\&A, 428, 1001

Coşkunoğlu, B., Ak, S., Bilir, S., et al. 2011, MNRAS, 412, 1237

Collier Cameron, A., Bouchy, F., Hébrard, G., et al. 2007, MNRAS, 375, 951

Collier Cameron, A., Guenther, E., Smalley, B., et al. 2010, MNRAS, 407, 507

Collins, K. A., Kielkopf, J. F., Stassun, K. G., \& Hessman, F. V. 2017, AJ, 153,77

Cutri, R. M., Skrutskie, M. F., van Dyk, S., et al. 2003, yCat, 2246, 0

Cutri, R. M., Wright, E. L., Conrow, T., et al. 2012, yCat, 2311, 0

Cutri, R. M., Wright, E. L., Conrow, T., et al. 2013, yCat, 2328, 0

Demarque, P., Woo, J.-H., Kim, Y.-C., \& Yi, S. K. 2004, ApJS, 155, 667

Demory, B.-O., \& Seager, S. 2011, ApJS, 197, 12

Donati, J.-F., Semel, M., Carter, B. D., Rees, D. E., \& Collier Cameron, A. 1997, MNRAS, 291, 658

Eastman, J., Gaudi, B. S., \& Agol, E. 2013, PASP, 125, 83

Eastman, J., Siverd, R., \& Gaudi, B. S. 2010, PASP, 122, 935

Fulton, B. J., Shporer, A., Winn, J. N., et al. 2011, AJ, 142, 84

Furlan, E., Ciardi, D. R., Everett, M. E., et al. 2017, AJ, 153, 71

Gaia Collaboration, Brown, A. G. A., Vallenari, A., et al. 2016, A\&A, in press, arXiv: 1609.04172

Gaudi, B. S., Stassun, K. G., Collins, K. A., et al. 2017, Natur, 546, 514

Grenier, S., Burnage, R., Faraggiana, R., et al. 1999, A\&AS, 135, 503

Hartman, J. D., Bakos, G. Á, Buchhave, L. A., et al. 2015, AJ, 150, 197

Hayward, T. L., Brandl, B., Pirger, B., et al. 2001, PASP, 113, 105

Henden, A. A., Levine, S., Terrell, D., \& Welch, D. L. 2015, in AAS Meeting 225 Abstracts, 336.16

Høg, E., Fabricius, C., Makarov, V. V., et al. 2000, A\&A, 355, L27

Hoyer, S., Pallé, E., Dragomir, D., \& Murgas, F. 2016, AJ, 151, 137

Husser, T.-O., Wende-von Berg, S., Dreizler, S., et al. 2013, A\&A, 553, A6

Iorio, L. 2011, Ap\&SS, 331, 485

Jensen, E. 2013, Tapir: A Web Interface for Transit/Eclipse Observability, Astrophysics Source Code Library, ascl:1306.007

Johnson, D. R. H., \& Soderblom, D. R. 1987, AJ, 93, 864

Johnson, M. C., Cochran, W. D., Albrecht, S., et al. 2014, ApJ, 790, 30

Kozai, Y. 1962, AJ, 67, 591

Kraft, R. P. 1967, ApJ, 150, 551

Kuhn, R. B., Rodriguez, J. E., Collins, K. A., et al. 2016, MNRAS, 459, 4281

Kurucz, R. L. 1992, in IAU Symp. 149, The Stellar Populations of Galaxies, ed. B. Barbuy \& A. Renzini (Dordrecht: Kluwer), 225

Latham, D. W., Bakos, G. Á, Torres, G., et al. 2009, ApJ, 704, 1107

Lidov, M. L. 1962, P\&SS, 9, 719

Lund, M. B., Rodriguez, J. E., Zhou, G., et al. 2017, arXiv:1707.01518 
Mamajek, E. E., \& Hillenbrand, L. A. 2008, ApJ, 687, 1264

Mandel, K., \& Agol, E. 2002, ApJL, 580, L171

Matsumura, S., Peale, S. J., \& Rasio, F. A. 2010, ApJ, 725, 1995

McCullough, P. R., Stys, J. E., Valenti, J. A., et al. 2006, ApJ, 648, 1228

Mortier, A., Santos, N. C., Sousa, S. G., et al. 2013, A\&A, 558, A106

Mortier, A., Sousa, S. G., Adibekyan, V. Z., Brandão, I. M., \& Santos, N. C. 2014, A\&A, 572, A95

Murray-Clay, R. A., Chiang, E. I., \& Murray, N. 2009, ApJ, 693, 23

Nelder, J. A., \& Mead, R. 1965, CompJ, 7, 308

Ogilvie, G. I. 2014, ARA\&A, 52, 171

Penev, K., Zhang, M., \& Jackson, B. 2014, PASP, 126, 553

Pepper, J., Gould, A., \& Depoy, D. L. 2003, AcA, 53, 213

Pepper, J., Kuhn, R. B., Siverd, R., James, D., \& Stassun, K. 2012, PASP, 124,230

Pepper, J., Pogge, R. W., DePoy, D. L., et al. 2007, PASP, 119, 923

Quinn, S. N., White, R. J., Latham, D. W., et al. 2014, ApJ, 787, 27

Richard, O., Michaud, G., \& Richer, J. 2001, ApJ, 558, 377

Richer, J., Michaud, G., \& Turcotte, S. 2000, ApJ, 529, 338

Schlegel, D. J., Finkbeiner, D. P., \& Davis, M. 1998, ApJ, 500, 525

Shporer, A., Jenkins, J. M., Rowe, J. F., et al. 2011, AJ, 142, 195
Siverd, R. J., Beatty, T. G., Pepper, J., et al. 2012, ApJ, 761, 123

Skrutskie, M. F., Cutri, R. M., Stiening, R., et al. 2006, AJ, 131, 1163

Stassun, K. G., \& Torres, G. 2016, ApJL, 831, L6

Swift, J. J., Bottom, M., Johnson, J. A., et al. 2015, JATIS, 1, 027002

Talens, G. J. J., Albrecht, S., Spronck, J. F. P., et al. 2017a, A\&A, in press, arXiv: 1707.04262

Talens, G. J. J., Justesen, A. B., Albrecht, S., et al. 2017b, A\&A, in press, arXiv: 1707.01500

Titus, J., \& Morgan, W. W. 1940, ApJ, 92, 256

Torres, G., Andersen, J., \& Giménez, A. 2010, A\&ARv, 18, 67

Tull, R. G., MacQueen, P. J., Sneden, C., \& Lambert, D. L. 1995, PASP, 107,251

van Leeuwen, F. 2007, A\&A, 474, 653

Winn, J. N., Fabrycky, D., Albrecht, S., \& Johnson, J. A. 2010, ApJL, 718, L145

Wright, E. L., Eisenhardt, P. R. M., Mainzer, A. K., et al. 2010, AJ, 140, 1868

Zhou, G., Latham, D. W., Bieryla, A., et al. 2016a, MNRAS, 460, 3376

Zhou, G., Rodriguez, J. E., Collins, K. A., et al. 2016b, AJ, 152, 136

Zucker, S., \& Mazeh, T. 1994, ApJ, 420, 806 